\title{
Impact of non-Gaussian beam profiles in the performance of hadron colliders
}

\author{
S. Papadopoulou® \\ European Organization for Nuclear Research (CERN), CH-1211 Geneva 23, Switzerland; \\ Department of Physics, University of Crete, P.O. Box 2208, GR-71003 Heraklion, Greece; \\ and Institute of Theoretical and Computational Physics (ITCP), GR-71003 Heraklion, Greece \\ F. Antoniou, T. Argyropoulos, M. Hostettler, Y. Papaphilippou®, and G. Trad \\ European Organization for Nuclear Research (CERN), CH-1211 Geneva 23, Switzerland
}

(Received 3 March 2020; accepted 12 October 2020; published 27 October 2020)

\begin{abstract}
At the Large Hadron Collider (LHC), the interplay between a series of effects, including intrabeam scattering (IBS), synchrotron radiation, longitudinal beam manipulations, two beam effects (beam-beam, e-cloud) and machine nonlinearities, can change the population of the core and tails and lead to nonGaussian beam distributions, at different periods during the beam cycle. By employing generalized distribution functions, it can be demonstrated that the modified non-Gaussian beam profiles have an impact in the beam emittance evolution by itself and thereby to the collider luminosity. This paper focuses on the estimation of beam distribution modification and the resulting rms beam size due to the combined effect of IBS and synchrotron radiation by employing a Monte-Carlo simulation code which is able to track 3D generalized particle distributions (SIRE). The code is first benchmarked over classical semianalytical IBS theories and then compared with measurements from the LHC at injection and collision energies, including projections for the High-Luminosity LHC (HL-LHC) high brightness regime. The impact of the distribution shape on the evolution of the bunch characteristics and machine performance is finally addressed.
\end{abstract}

DOI: 10.1103/PhysRevAccelBeams.23.101004

\section{INTRODUCTION}

The performance of a high-energy hadron collider such as the LHC is heavily based on the preservation of the injected emittances, under the influence of several degrading mechanisms. In this respect, an emittance evolution model was constructed including the effects of intrabeam scattering (IBS), synchrotron radiation, elastic scattering and luminosity burn-off (while at collision) [1]. This simple model is based on semianalytical approaches which assume Gaussian beam distributions, in particular for IBS. The bunch characteristics evolution predicted by this model revealed discrepancies, as compared to the measurements, translated to differences in the luminosity predicted by the model as compared to the experimental estimations [1-3]. One of the possible reasons for these differences could be attributed to the fact that the bunch profiles appear to be non-Gaussian both at injection and collision energies, i.e.,

Published by the American Physical Society under the terms of the Creative Commons Attribution 4.0 International license. Further distribution of this work must maintain attribution to the author(s) and the published article's title, journal citation, and DOI.
$450 \mathrm{GeV}$ and $6.5 \mathrm{TeV}$ respectively. The aim of this study is to quantify the impact of the beam distribution shape on the emittance and luminosity evolution of hadron colliders. In order to illustrate this, and employing generalized distribution functions, the luminosity of non-Gaussian beams is determined in a closed form. The generalization of the luminosity estimate for arbitrary distributions does not only permit its comparison to the usual Gaussian beam estimate but also the extension of classical results for the impact of non-Gaussian beam distributions to the luminosity (see Sec. II). This motivates the investigation of the emittance evolution beyond the classical analytical formulas for modeling IBS, which are based on 3D Gaussian beam assumptions [4]. In this respect, a Monte Carlo multiparticle simulation code for IBS and Radiation Effects (SIRE) [5,6], is employed and compared to LHC data from Run2. A brief description of the code, and its benchmarking with the existing IBS analytical approaches and simulations for lepton rings is presented in Sec. III. Extending previous benchmarking studies for the LHC with respect to IBS theories [7], a detailed comparison of the Bjorken-Mtingwa (B-M) IBS theoretical model with the SIRE code for both injection and collision energies is presented for the nominal LHC using the batch 
compression merging and splitting (BCMS) $[8,9]$ beam and the high luminosity LHC (HL-LHC) [10] beam parameters (Sec. IV). Finally, measured data from the LHC corresponding to non-Gaussian longitudinal beam profiles are compared with the expectations of the rms emittance evolution given by the theoretical B-M analytical formalism [4] and the SIRE code (Sec. V).

\section{MOTIVATION-IMPACT OF NON-GAUSSIAN DISTRIBUTIONS ON LUMINOSITY}

The performance of a collider is determined by the luminosity which, for two beams colliding head-on, is given by [11]:

$$
\begin{aligned}
\mathcal{L}= & 2 N_{1} N_{2} N_{b} f_{\text {rev }} \iiint \int_{-\infty}^{\infty} \rho_{1 x}(x) \rho_{1 y}(y) \rho_{1 s}\left(s-s_{0}\right) \\
& \times \rho_{2 x}(x) \rho_{2 y}(y) \rho_{2 s}\left(s+s_{0}\right) d x d y d s d s_{0},
\end{aligned}
$$

with $N_{1,2}$ representing the number of particles per bunch for each beam, $N_{b}$ the total number of colliding bunches, $f_{\text {rev }}$ the revolution frequency and $\rho$ the beam density distribution functions for each plane and beam.

Based on Eq. (1), assuming Gaussian beams that collide head-on, the luminosity is expressed as [11]:

$$
\mathcal{L}^{G}=\frac{N_{1} N_{2} N_{b} f_{\text {rev }}}{4 \pi \sigma_{x}^{G} \sigma_{y}^{G}} .
$$

In order to achieve high luminosity, high intensity bunches and small beam sizes are required. The horizontal and vertical beam sizes of two colliding Gaussian bunches are given by:

$$
\sigma_{x}^{G}=\sqrt{\frac{\sigma_{1 x}^{2}+\sigma_{2 x}^{2}}{2}} \text { and } \sigma_{y}^{G}=\sqrt{\frac{\sigma_{1 y}^{2}+\sigma_{2 y}^{2}}{2}},
$$

where $\left(\sigma_{1 x}, \sigma_{1 y}\right)$ and $\left(\sigma_{2 x}, \sigma_{2 y}\right)$ are the transverse rms beam sizes of beam 1 and beam 2 , respectively.

Based on the transverse and longitudinal bunch profile measurements, it has been observed that the particle distributions in the LHC, both at collision and injection energies, appear to have shapes that differ from the ones of a normal distribution [12-14]. At the LHC injection energy, the emittance evolution is dominated by the IBS effect, both in the horizontal and in the longitudinal plane, while no effect is expected in the vertical plane [15] where dispersion is minor. From Run 2 data, it is observed that in many cases the transverse bunch profiles appear to be nonGaussian during the whole injection plateau [12]. At the LHC collision energy, the IBS effect is weaker, while synchrotron radiation damping becomes more pronounced. The bunch profiles at collisions appear to have nonGaussian tails, as well. In fact, during the energy ramp, the bunches that are blown up longitudinally in order to avoid instabilities due to the loss of Landau damping [16], arrive at the start of collisions with a clearly non-Gaussian shape [14].

By assuming that a particle distribution is Gaussian when this is not the case, not only the rms beam size may be underestimated or overestimated, but also its impact on performance parameters, such as the luminosity. Therefore, it is important to use appropriate fitting functions (or some type of interpolation algorithm) on the beam profile in order to properly address this discrepancy. A generalized Gaussian function, called the $q$-Gaussian [17], can be employed for fitting more accurately bunch profiles with shapes that differ from the ones of a normal distribution (see the Appendix A for the properties of this distribution function). The parameter $q$ describes the weight of the tails as compared to the core, ranging from light tailed ones for $q<1$ (including the square distribution for $q \rightarrow-\infty$ ) and extending to a heavy tailed ones for $q>1$, passing through the Gaussian distributions in the limit of $q \rightarrow 1$. This distribution is actually a stationary solution of a generalized Fokker-Plank equation which can cover a full spectrum of statistical behaviors of dynamical systems, from sub to super-diffusion Levy-type processes [18].

In view of quantifying the impact of non-Gaussian distributions, the luminosity is estimated through Eq. (1) by using the specified probability density functions. Assuming that the two beams are identical and that they collide head-on, the luminosity for $q$-Gaussian distribution functions in the transverse plane is given by:

$$
\mathcal{L}^{q G}=\frac{N_{1} N_{2} N_{b} f_{\text {rev }}}{4 \pi \sigma_{x}^{q G} \sigma_{y}^{q G}} \mathcal{I}_{x}^{q G} \mathcal{I}_{y}^{q G},
$$

for $\sigma_{x, y}^{q G}$ being the rms beam sizes (see Appendix A) in the transverse plane, for both beams. The factors $\mathcal{I}_{x, y}^{q G}$ which depend on the parameter $q$ in the respective planes and the details of the calculation are presented in Appendix B, together with the validation of the luminosity estimation for $q$-Gaussian distributions (shown in Fig. 22). By comparing this equation to the standard luminosity formula for Gaussian beams with identical rms sizes, the significance of the tail population contribution on luminosity can be established and parametrized through $q$. This is illustrated in Fig. 1 where the luminosity variation normalized to the corresponding one for Gaussian beams $\left(\mathcal{L}^{G}\right)$ is parametrized with the parameter $q$ of the $q$-Gaussian fitting function, characterizing the weight of the tails in the transverse plane, for fixed $q$-Gaussian rms beam sizes in all planes, assuming head on collisions (i.e., no dependence of the luminosity on the longitudinal beam size, see Appendix B). The bunch profiles corresponding to a light tailed $(q<1)$, a Gaussian $(q=1)$ and a heavy tailed $(q>1)$ distribution, having identical beam sizes, are plotted in Fig. 2. As $q$ (and $\beta^{q G}$ that is a parameter of the q-Gaussian probability density function and a real 


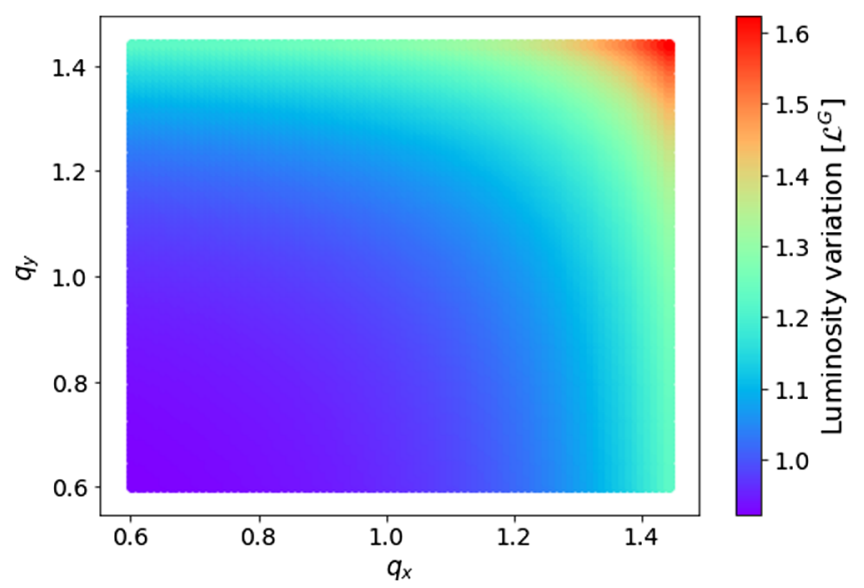

FIG. 1. Parameterization of the luminosity variation, normalized to the corresponding Gaussian luminosity value $\mathcal{L}^{G}$, with the weight of the transverse distribution tails given by the parameters $q_{x, y}$, for a constant q-Gaussian rms beam size.

positive number-see Appendix A) vary for fixed $q$-Gaussian $\mathrm{rms}$ beam sizes (based on Eq. (A5) in Appendix A), the luminosity varies as well with respect to the one estimated for purely Gaussian beams. Practically, if the tails of a distribution differ by $10 \%$ compared to the ones of a Gaussian distribution (i.e., $q=0.9$ or $q=1.1$ ), the luminosity value can be overestimated or underestimated by $5 \%$. It is then clear that, for two beams colliding head-on, the shape of the transverse distributions has a significant impact to the estimated luminosity, in particular for the LHC experiments which target a 2\% accuracy in their estimates [19]. The impact of nonGaussian distributions on the luminosity was firstly discussed by Hereward [20]. In particular, the luminosity integrals were calculated for several distributions as the rectangular and the parabolic which correspond to a $q$-Gaussian with $q \rightarrow-\infty$ and $q=0$, respectively. Assuming that the two beams are identical and that they collide head-on, these distributions were used as examples to estimate the discrepancy from the luminosity for Gaussian densities. This discrepancy is calculated after solving the $\mathcal{L}_{w}$ that is the integral over the density functions in one plane, being identical for both beams, as:

$$
\mathcal{L}_{w}\left\langle w^{2}\right\rangle^{1 / 2}=\sigma_{w} \int_{-\infty}^{\infty} \rho(w)^{2} d w,
$$

with $w=x, y$ and for $\sigma_{w}$ being the transverse rms beam size, since these solutions correspond to the transverse plane. It was found that $[20,21]$ :

$\mathcal{L}_{w}\left\langle w^{2}\right\rangle^{1 / 2}= \begin{cases}0.2887, & \text { for a rectangular distribution } \\ 0.2683, & \text { for a parabolic distribution } \\ \frac{1}{2 \sqrt{\pi}}=0.2821, & \text { for a Gaussian distribution }\end{cases}$

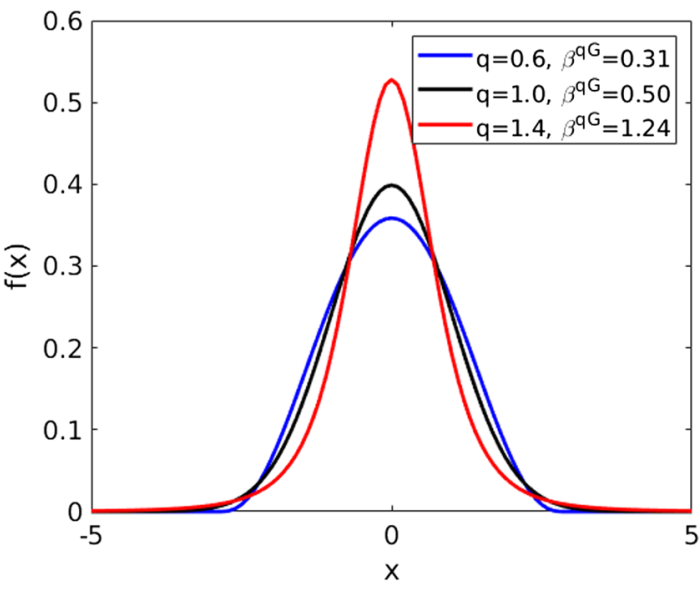

FIG. 2. The $q$-Gaussian density distribution function for a light tailed (blue, $q<1$ ), a Gaussian (black, $q=1$ ) and a heavy tailed (red, $q>1$ ) bunch profile, having identical $q$-Gaussian rms beam sizes.

In fact, this approach already identified the existence of a minimum for a light tailed parabolic distribution, which becomes obvious by employing the $q$-Gaussian, as observed in Fig. 3, where $\mathcal{L}_{w}\left\langle w^{2}\right\rangle^{1 / 2}$ is plotted versus $q$ for $q<1$, i.e., light tails (left), and $q>1$, i.e., heavy tails (right). The results for $q$-Gaussian distributions (grey curves) are in perfect agreement with the case studies discussed in [20]. This is also true for a rectangular distribution which corresponds to a $q$-Gaussian with $q \rightarrow-\infty$ and is beyond the range of the left plot of Fig. 3. For heavy tailed distributions, there is no upper limit for the constant of Eq. (6), as already inferred by Hereward [20]. In Fig. 3 (right) the case of a heavy tailed distribution with $q=1.65$ is denoted by a red square. The extreme case of $q \rightarrow 5 / 3$ corresponds to a $q$-Gaussian whose rms size goes to infinity (i.e., Levy distributions, see Appendix A).

The sensitivity of the luminosity on the distribution as generalized by employing the $q$-Gaussian function justifies the need of carefully studying the evolution of distributions in hadron colliders. For the LHC, a luminosity model was constructed [1]. The evolution of the emittance includes the effects of IBS, synchrotron radiation, elastic scattering, betatron coupling, noise and burn-off. Although this model has a relative agreement with respect to the measured luminosities by the experiments (i.e., ATLAS [22] and CMS [23]), there is still some room for improvement [3]. Indeed, the emittance evolution for IBS was based on the module of MAD-X [24] following the B-M theory which assumes Gaussian beam distributions. The extension of this to non-Gaussian distributions as observed in the LHC may shed light to the origin of the remaining discrepancy between the model and the measurements. 

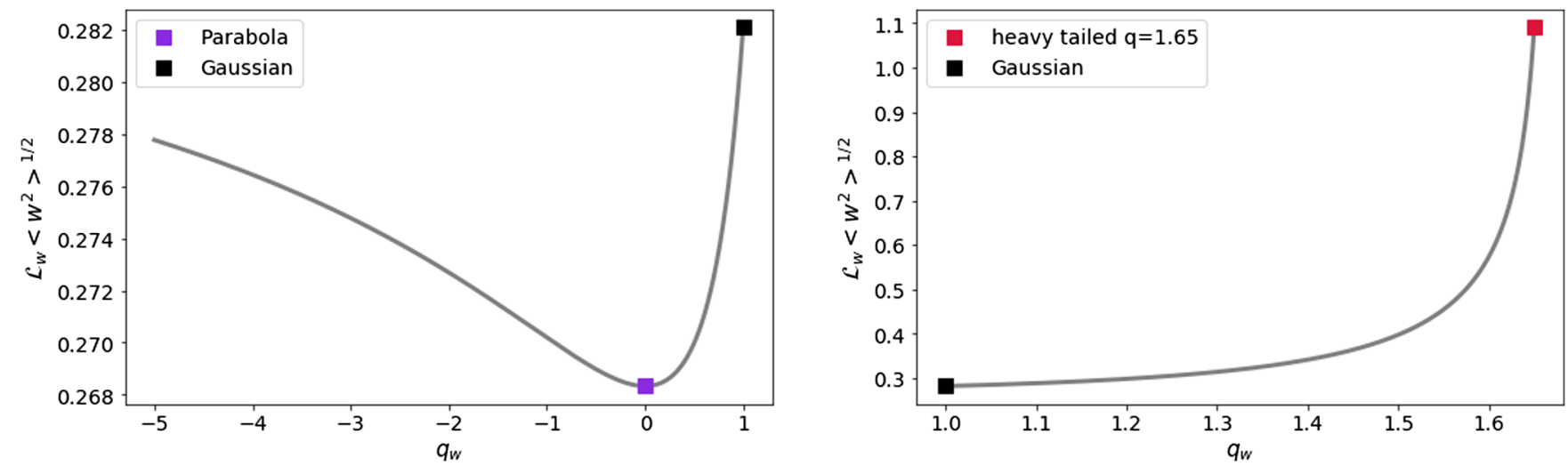

FIG. 3. In order to estimate the divergence of the luminosity for non-Gaussian distributions from the one for Gaussian densities, the $\mathcal{L}_{w}\left\langle w^{2}\right\rangle^{1 / 2}$ [see Eq. (5)] is plotted versus the weight of the tails $q$, for light tailed (left) and heavy tailed (right) distributions, i.e., $q<1$ and $q>1$, respectively. The points corresponding to a parabola and a Gaussian distribution, firstly presented in $[20,21]$, are plotted.

\section{INTRABEAM SCATTERING THEORIES, OBSERVATIONS, AND SIMULATIONS}

One of the statistical processes causing a spreading of particles in phase space or a continuous increase of beam emittance is the small angle multiple Coulomb scattering, called intrabeam scattering (IBS), which plays an important role in $e^{+} / e^{-}$damping rings, high intensity/low energy light sources [25] and high intensity hadron [26] and ion [27] circular machines. The IBS theory for accelerators was firstly introduced by Piwinski [28] and extended by Martini [29], establishing a formulation called the standard Piwinski method. Later, Bjorken and Mtingwa (BM) [4] used a different approach to describe the effect, taking into account the strong focusing effect. The modified Piwinski method [30] that includes the strong focusing effect, was developed by Bane. Some approximations of these theories are the high energy one by Bane [30] and the completely integrated modified Piwinski [31]. A different approach developed by Lebedev for hadron beams is based on a Boltzmann type integro-differential equation and includes betatron coupling [32].

The analytical models that describe the IBS effect $[4,33]$ assume Gaussian beam distributions. The stationary solution of the Fokker-Planck equation is a particle distribution that is Gaussian in the phase space. However, taking into account the effects of IBS, radiation damping and quantum excitation but also other diffusive mechanisms [34], there is no evidence that the distribution remains Gaussian. Therefore, it is important to develop analytical formulas and simulation tools that calculate the interplay between these effects for any distribution.

For the Relativistic Heavy Ion Collider (RHIC), the IBS growth rates were calculated and benchmarked with experimental data using the distribution function evolution (based on the Fokker-Planck equations), extending the usual approach of employing a conventional Gaussian-like distribution [35]. In this respect, IBS growth rates were calculated for a bi-gaussian distribution, which was interesting for studying the possibility of using electron cooling in RHIC [36]. Later, a model which is suitable for IBS calculations for arbitrary distribution functions and its comparison to experimental data was presented in [37]. The IBS effect was also studied for high-brightness electron linac beams which appear to be non-Gaussian, especially in the longitudinal plane [38]. For low-emittance high-intensity electron storage rings, the interplay between intrabeam scattering and wake-field forces is discussed in [39]. In particular, the calculation of the IBS growth rates and the estimation of the emittance growth is discussed in detail in [40], where the importance of knowing the formation of the distribution tails is underlined, referring also to the use of Monte-Carlo methods.

In order to simulate the impact of a distribution shape on the emittance evolution when considering IBS and radiation effects the SIRE (software for IBS and radiation effects) $[5,6]$ code was developed by Vivoli and Martini at CERN. A similar Monte Carlo approach (IBStrack [41]) was implemented also in the collective effects simulation tool CMAD $[42,43]$. Both algorithms have as their basis MOCAC (MOnte CArlo Code), a Monte-Carlo code developed by Zenkevich and collaborators [44,45], which calculates the IBS effect for arbitrary distributions, by representing the beam through a large number of macroparticles occupying points in the 6-dimensional phase space. Being an extension of MOCAC, SIRE was developed to simulate the evolution of the beam particle distributions, taking into account the effects of IBS, synchrotron radiation and quantum excitation. For the evolution of the LHC bunch parameters and the shape of the bunch profiles presented in this paper, the simulations are performed using SIRE. As the physics and implementation details of the code are extensively explained in [6], we only briefly summarizes some of its key features. 


\section{A. Software for IBS and radiations effects (SIRE)}

For the IBS calculations, SIRE [5,6] uses the classical Rutherford cross section, which is closer to the Piwinski formalism [33]. It uses as input the optics functions at different locations of the lattice in order to determine the trajectories of the particles in phase space. Instead of using the 6 coordinates for position and momentum, the two Courant-Snyder and longitudinal invariants and the 3 phases (betatron and synchrotron) are used. For a linear ring, the 3 invariants are conserved between points around the lattice and can only be changed by the effects of IBS, synchrotron radiation and quantum excitation, while the phases are chosen randomly at each given point of the lattice. The time steps for which the IBS and radiation effects are called should be specified such that they are larger than the revolution time and smaller than the damping/growth times. Dividing the total time by the time steps shows how frequently the IBS, synchrotron radiation and quantum excitation routines are called. The quantum excitation is implemented by adding to the 6 coordinates of each macroparticle a Gaussian random noise component.

Depending on the elapsed time, the synchrotron radiation damping acts on the invariants of the macroparticles as an exponential decrement. The routine introduced for this reason is called after the calculation of the IBS effect at each iteration. Using small iteration time steps $d t$ (which are much smaller than the damping times and for which the emittances change adiabatically), the evolution of the transverse emittance and energy spread due to the effects of IBS and synchrotron radiation can be obtained by solving the coupled differential equations:

$$
\begin{aligned}
\frac{d \epsilon_{x, y}}{d t} & =\frac{-2\left(\epsilon_{x, y}-\epsilon_{x, y 0}\right)}{\tau_{x, y}}+\frac{2 \epsilon_{x, y}}{T_{x, y}}, \\
\frac{d \sigma_{p}}{d t} & =\frac{-\left(\sigma_{p}-\sigma_{p 0}\right)}{\tau_{p}}+\frac{\sigma_{p}}{T_{p}},
\end{aligned}
$$

with $\epsilon_{x, y 0}$ and $\sigma_{p 0}$ being the zero current (without the effect of IBS) equilibrium transverse emittances and energy spread, respectively. The $\tau_{x, y}, \tau_{p}$ are the synchrotron radiation damping times and $T_{x, y}, T_{p}$ the IBS growth times.

The algorithm SIRE uses to calculate IBS is similar to that implemented in MOCAC, where the beam is represented by a large number of macroparticles occupying points in the 6-dimensional phase space. The default distribution defined in SIRE by using a random number generator, is the Gaussian and is given in action angle variables. In order to introduce a different distribution, the proper random deviates should be generated or the action angle variables of all macroparticles for the desired distribution should be provided. After specifying the total beam population and the number of macroparticles, the initial distribution can be tracked. IBS depends strongly on beam brightness, whereas synchrotron radiation is independent, thereby radiation damping is uniform through the whole beam distribution. In this respect, the core of the distribution is blown-up by IBS (in particular in the horizontal and longitudinal plane), whereas the tails are getting reduced. This is exactly what is demonstrated with the simulation results presented in the following section, where the core of the distributions appears to blow up in such a way that it covers up the tails, resulting in distributions that become light tailed. The particle distribution in all planes can be saved as often as requested during the simulation time. Currently, the output file gives the evolution of the emittance in all planes for the specified time steps.

After providing the beam distribution and the optics along a lattice, the beam is geometrically divided according to the specified number of cells for each plane. The macroparticles are assigned to each cell according to their geometrical position. For each lattice point defined in the optics file, the 3 phases of each macroparticle are randomly chosen and the position and momentum of the macroparticles are calculated. Based on the classical Rutherford cross section, intrabeam collisions between pairs of macroparticles are calculated in each cell. The momentum of particles is changed due to scattering. The number of macroparticles and cells, i.e., the number of collisions each macroparticle experiences, is chosen so as to give accurate results for a reasonable computational time. The scattering angles for each collision are determined. In order to get the mean value of the emittance and momentum deviation changes for all particles, we have to integrate over the phase space volume of betatron coordinates, momentum deviations and azimuthal positions of the interacting particles. The beam distribution is then updated based on the new invariants of the macroparticles. For a specified number of time steps which practically shows how frequently the IBS and synchrotron radiation routines are called, the beam distribution is updated and the rms beam emittances are recomputed, giving finally as output the emittance evolution in time. The simulation proceeds to the next lattice point and continues until the end time is reached.

A lattice compression technique named "lattice recurrences" has been implemented to speed up the calculations [5]. Since the increase of the invariants due to IBS is linear to the first order in the traveling time along an element, the IBS kicks with optics functions differing less than a specified precision value are considered equivalent. For the corresponding group of elements, the IBS effect is evaluated only once, resulting to a reduction of the computational time.

\section{B. The logarithmic Coulomb factor}

The IBS growth times have a complicated dependence on the beam properties, due to the coupling of the three planes through dispersion. Some of these properties are the bunch charge and energy, the beam optics and the equilibrium rms horizontal, vertical emittances and the energy 
spread. The IBS growth times depend also on a logarithmic Coulomb factor which is used to include the contribution of events having a very large and very small impact parameter. The typical way of computing a log factor overemphasizes the importance of the very small impact parameter events, for which the tails of the steady-state bunch distributions are non-Gaussian. In the high energy approximation by Bane [25], in order to describe the size of the core of the bunch, the Coulomb log factor is calculated as was first proposed by Raubenheimer [46], i.e., based on a boundary between the contribution to the core and the tails. In B-M, Bane and CIMP methods, the Coulomb factor is defined as the ratio of the maximum $r_{\max }$ to the minimum $r_{\min }$ impact parameter in the collision of two particles in the bunch, that is $(\log ) \equiv \ln \left(r_{\max } / r_{\min }\right)$. For typical flat beams, the $r_{\max }$ is taken to be equal to the vertical beam size $\sigma_{y}$, while $r_{\min }$ is taken to be $r_{\min }=r_{0} \beta_{x} /\left(\gamma^{2} \epsilon_{x}\right)$, with $r_{0}$ being the classical particle radius. Then, the Coulomb factor can be written as:

$$
(\log )=\ln \left(\frac{\gamma^{2} \epsilon_{x} \sqrt{\beta_{y} \epsilon_{y}}}{r_{0} \beta_{x}}\right) .
$$

The formalism by Piwinski always seems to underestimate the IBS effect with respect to the other theoretical models. What diversifies Piwinski's method, is the different definition of the Coulomb factor. In that approach, the maximum impact parameter which is typically taken as the vertical beam size appears. In the high energy limit, with $d$ being the maximum impact parameter, the Coulomb $(\log )$ for Piwinski can be written as [47]:

$$
(\log )=\ln \left(\frac{d \sigma_{x}^{2}}{4 r_{0} a^{2}}\right)
$$

where $a=\frac{\sigma_{x}}{\gamma} \sqrt{\frac{\beta_{x}}{\epsilon_{x}}}$. Comparing the $(\log )$ factors of Eq. (8) and (9), we find that condition $d=4 \sigma_{y}$ in order for the Piwinski approach agreeing with the other models. SIRE uses the "binary collision map" algorithm, conceived by Zenkevich, which allows to reduce the effects of the continuous time dynamical IBS system to a discrete time map in momentum space. For the binary collision events, the maximum impact parameter is taken as the beam height.

\section{Benchmarking of IBS theoretical model with SIRE}

The performance of hadron machines is limited by the IBS effect causing emittance growth. For lepton machines such as future linear collider damping rings, new generation light sources and B-factories, the IBS effect can also be predominant. It is thus important to study the IBS theories in the presence of synchrotron radiation and quantum excitation and benchmark the existing theoretical models and tracking codes with experimental data. In this way, the codes limitations can be identified so that to apply the necessary improvements in order to get better predictions for a machine's operation.

A benchmarking of the IBS theoretical models with Monte-Carlo codes is presented in [48] for lepton rings. The comparison between different theoretical models and SIRE is discussed for the Compact Linear Collider (CLIC) damping ring (DR) [49], having ultralow emittances which are strongly dominated by IBS, in the presence of synchrotron radiation and quantum excitation. Results of this comparison are presented in Fig. 4, for one turn of the DR lattice. Due to the fact that in SIRE the generation of the distribution is based on a random number generator, the tracking simulations were performed several times, resulting in the one standard deviation error bars (plotted in green). The classical formalism of Piwinski (red colored) and SIRE are in perfect agreement, as was expected, since SIRE uses the classical Rutherford cross section which is closer to the Piwinski formalism. The Bjorken-Mtingwa (black colored) and Bane (magenta colored) formalisms overestimate the effect compared to Piwinski method mainly due to a mismatch of the Coulomb factor used in the different approaches [see Eq. (9)].

The IBS theoretical models have been studied in detail and benchmarked with experimental data also for hadron beams over the years $[26,27]$. A comparison of the LHC
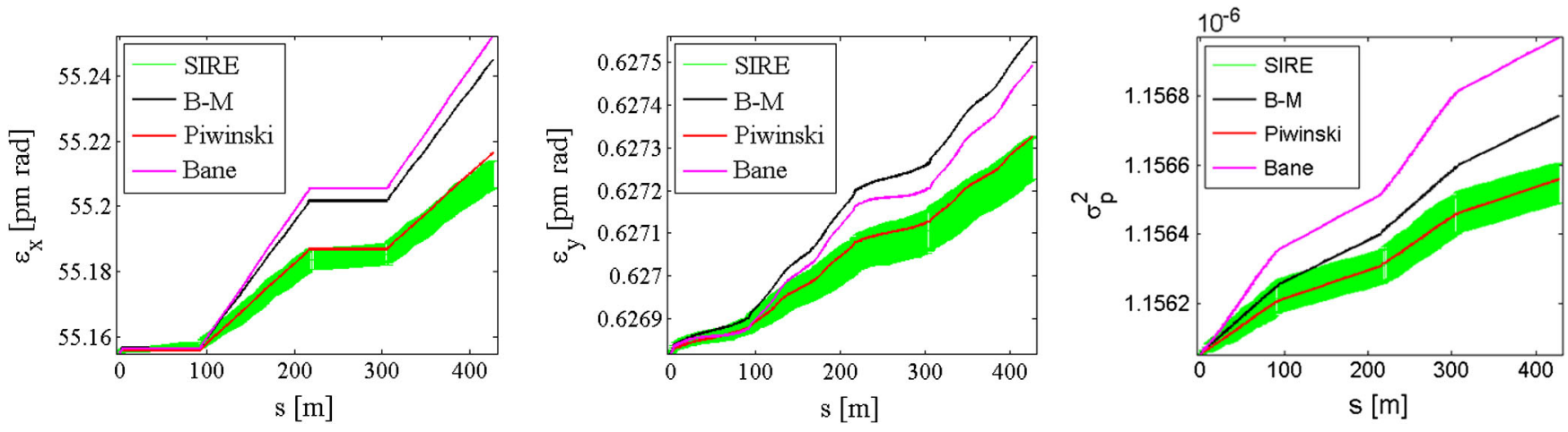

FIG. 4. One turn comparison for the horizontal (left) and vertical (middle) emittance and energy spread (right) between the tracking code SIRE and the theoretical models Bjorken-Mtingwa (B-M), Piwinski and Bane for the CLIC DR lattice. 
data with simulations performed with SIRE is discussed in $[7,13]$. In this paper, the SIRE simulations, as well as the benchmarking with the B-M formalism and experimental data are discussed in detail for the LHC and the High Luminosity LHC (HL-LHC) [10].

\section{SIMULATIONS PERFORMED WITH SIRE FOR THE LHC}

In order to understand the evolution of the bunch characteristics, based on the bunch profile observations, it is important to study the interplay between IBS and radiation effects (synchrotron radiation and quantum excitation) during the full LHC cycle. This is done using SIRE for two cases which are important for the current and future machine performance; the nominal BCMS [8,9] and the HL-LHC [10] parameters. Despite some blow-up in the LHC during the ramp, it is observed that the BCMS beam gives an increase in peak luminosity of around $20 \%$. The HL-LHC is the major LHC upgrade aiming to increase integrated luminosity by at least a factor of 10 compared to the nominal LHC design value (from 300 to $3000 \mathrm{fb}^{-1}$ ). In order to achieve that, the bunch population needs to be increased and the transverse beam size at the collision points has to be lowered.

Apart from the IBS and synchrotron radiation which are the dominant effects for the emittance evolution in the LHC, a combination of other diffusion mechanisms, like the beam-beam effect, electron-cloud, noise (due to the power converters, the transverse damper, the crab cavities, etc.), and other nonlinearities cause emittance growth and/ or particle losses [50]. Despite the fact that these mechanisms are not included in SIRE, it is possible to add empirically (i.e., based on observations) their contribution. Practically, there is the option of adding or complementing the variation of the bunch parameters in time. In fact, the simulation studies presented in this paper for the LHC are focused on the $3 \sigma$ range of the particle distributions and therefore, mechanisms which concern the far tail regime are not taken into account as they are more important for the lifetime of the beam then on distribution evolution.

\section{A. Reduced lattice}

As mentioned earlier, one of the inputs required by SIRE are the optical functions along the ring. As the LHC is a very long accelerator of about $27 \mathrm{~km}$, with a very large number of elements in the sequence (more than 11000), SIRE requires an extremely long computational time to track the distribution for all the elements along the ring. Aiming to reduce the computational time, a study was first performed in order to identify the optimal minimum number of critical IBS kicks around the lattice, without affecting the overall effect. The IBS growth rates were firstly calculated for the full optics of the LHC, using the IBS module of the Methodical Accelerator Design code (MAD-X) [24] which is based on the Bjorken-Mtingwa formalism. Figure 5 shows the IBS growth rates in the longitudinal (green), the horizontal (blue) and the vertical (magenta) plane. Taking into account the strong IBS kicks along the ring, various lattices with a reduced number of elements, including the case of the smooth lattice approximation, were tested. Then, using the IBS module of MAD-X, the emittance evolution was calculated for several sets of beam parameters to assure that the choice of the elements is valid for a wide range of regimes, for which the IBS impact may be weaker or stronger. Finally, the optimal lattice chosen consists of only 92 elements whose positions are denoted by red dashed lines in Fig. 5.

Figure 6 shows the emittance (left) and the bunch length (right) growth during $30 \mathrm{~min}$ at injection energy, for the nominal BCMS beams, with initial emittance and $4 \sigma$ bunch length that are respectively $\epsilon_{x 0}=1.5 \mu \mathrm{mrad}$ and $\sigma_{s 0}=1 \mathrm{~ns}$, having a bunch population of $1.2 \times 10^{11}$ protons. The black

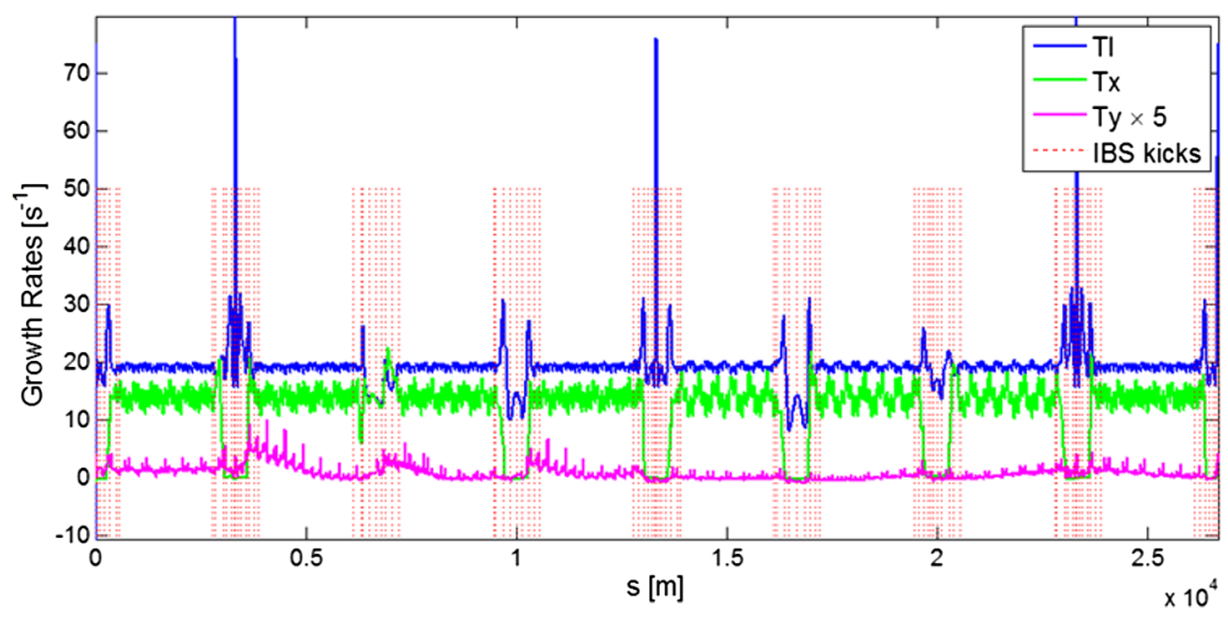

FIG. 5. The IBS growth rates along the LHC in all three planes: longitudinal (green), the horizontal (blue), and the vertical (magenta). The IBS kicks that are noted with red dashed lines, represent the positions of the 92 elements that compose the reduced lattice. 

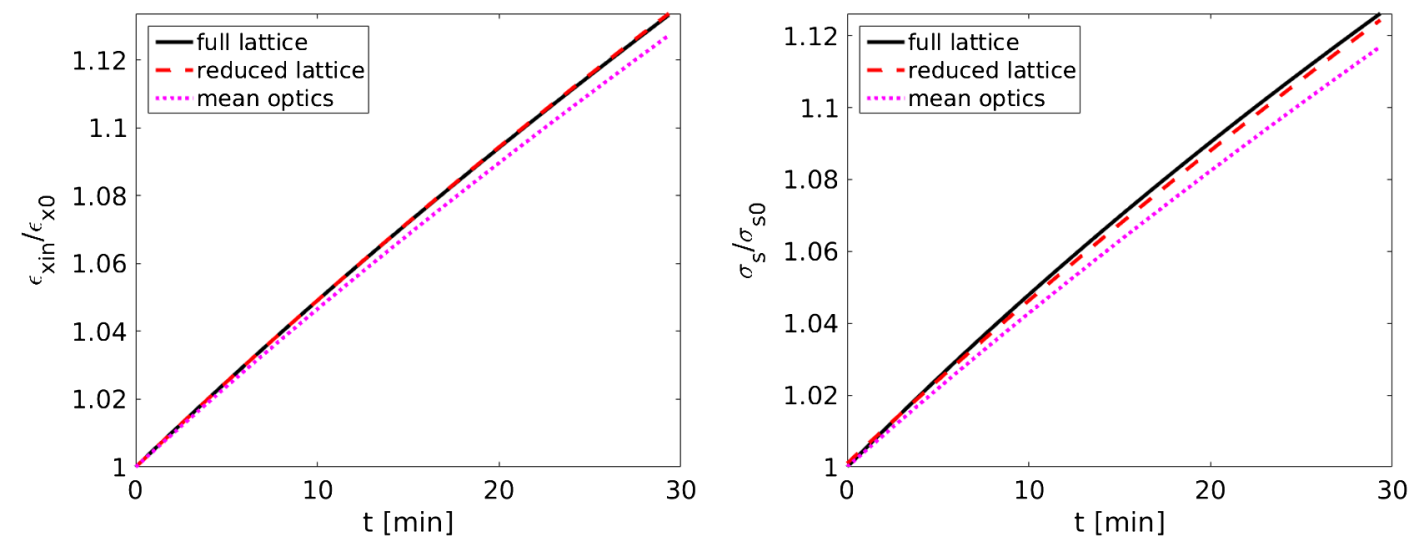

FIG. 6. The growth of the horizontal emittance (left) and bunch length (right) due to IBS, as computed by MAD-X with the BjorkenMtingwa formalism, in a time period of $30 \mathrm{~min}$ at injection, when considering the full lattice (black solid line), the reduced lattice (red dashed line) and the smooth lattice approximation- mean optics (magenta dotted line).

solid line refers to the case of the full lattice, while the red dashed one to the reduced lattice with the 92 elements. The magenta dotted line corresponds to the case of the smooth lattice approximation for which a lattice with a unique element, having the optics that represent in the best possible way the mean optics of the full lattice, is considered. The agreement of the full and the reduced lattice is very good in all planes. On the other hand, by using the smooth lattice approximation the IBS effect is slightly underestimated, in particular, in the longitudinal plane. In the next, since the results for the reduced and the full lattice agree also in SIRE, the reduced lattice is used as an input for the simulation code. After choosing the optimal number of cells and macroparticles, the computational time in the case of the reduced lattice is almost 20 times shorter than the one of the full LHC lattice.

\section{B. Convergence studies}

For a specified set of input beam parameters, various scans should be performed for different combinations of number of macroparticles and cells in order to find the optimal values which provide a fast tracking and at the same time, guarantee that the scattering process leads to accurate results. In these terms, in order to avoid having a very small number of macroparticles per cell, the total number of cells is calculated based on the optimal minimum number of macroparticles per cell. For $n_{x}, n_{y}$, $n_{z}$ being the number of cells in the horizontal, vertical and longitudinal plane, respectively, it is assumed that in the transverse plane there is a correlation between the number of cells ratio and the beam sizes ratio, meaning that $n_{x} / n_{y}=\sigma_{x} / \sigma_{y}$. Therefore, for $n_{m p}$ being the total number of macroparticles, the number of macroparticles per cell is:

$$
n_{m p} / \text { cell }=\frac{n_{m p}}{n_{x} n_{y} n_{z}}=\frac{n_{m p}}{n_{x}^{2}\left(\frac{\sigma_{y}}{\sigma_{x}}\right) n_{z}} .
$$

A scanning of the total number of cells is performed for an example set of beam parameters to be used as an input for tracking. Based on Eq. (10), by keeping the total number of macroparticles constant, the different combinations of cell numbers determines the number of macroparticles per cell. Figure 7 (left) shows the dependence of the emittance variation (ratio of final versus initial value) in the horizontal (blue) and longitudinal (green) plane on the number of macroparticles per cell, for a specified time
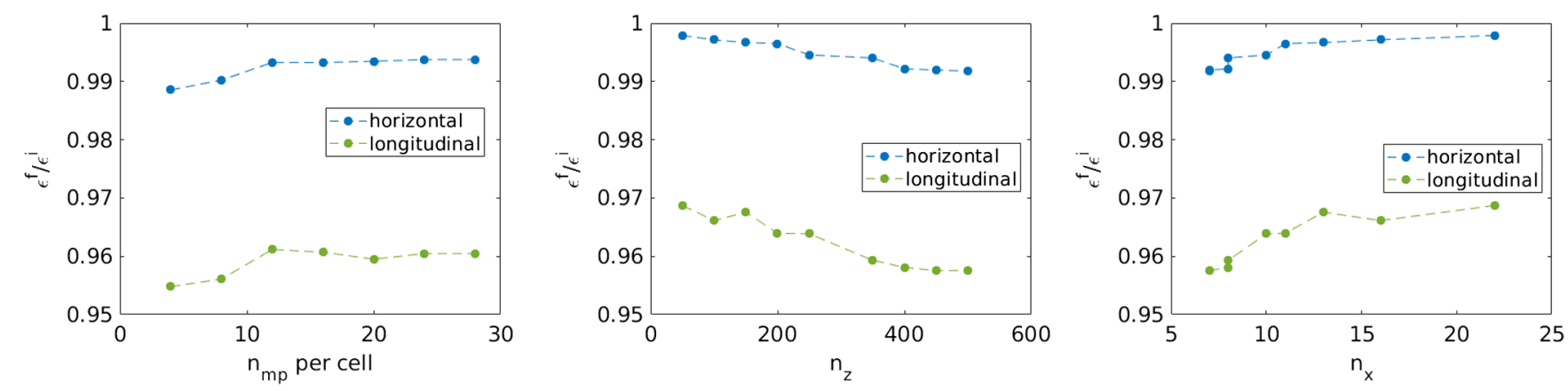

FIG. 7. The dependence of the horizontal (blue) and longitudinal (green) emittance variation on the number of macroparticles per cell (left), on the number of cells in the longitudinal plane (middle) and in the horizontal plane (right), for a specific time period. 
TABLE I. Nominal (BCMS) and HL-LHC parameters, at injection energy $(450 \mathrm{GeV})$ and at collision energy $(6.5 \mathrm{TeV})$.

\begin{tabular}{|c|c|c|c|c|}
\hline \multirow[b]{2}{*}{ IBS growths } & \multicolumn{2}{|c|}{ Injection energy } & \multicolumn{2}{|c|}{ Collision energy } \\
\hline & LHC (BCMS) & HL-LHC & LHC (BCMS) & HL-LHC \\
\hline$\epsilon_{x, y}[\mu \mathrm{m} \mathrm{rad}]$ & 1.5 & 2.0 & 2.5 & 2.5 \\
\hline $4 \sigma$ bunch length [ns] & 1.0 & 1.2 & 1.0 & 1.2 \\
\hline Bunch population $\left[10^{11}\right]$ & 1.2 & 2.3 & 1.1 & 2.2 \\
\hline
\end{tabular}

duration. The value of the number of macroparticles per cell after which the variation of the emittances in both planes remains constant is chosen as the optimal minimum value. After specifying this value, a scanning is performed for a fixed number of macroparticles, in order to choose the number of cells to be used, firstly in the longitudinal plane.

Then, the number of cells in the horizontal plane can be calculated using Eq. (10), when knowing the ratio of the beam sizes in the transverse plane. ${ }^{1}$ In Fig. 7, the dependence of the emittances variation is plotted versus the number of cells in the longitudinal (middle) and horizontal (right) plane, for a specific time duration. It can be noticed that the variation of the emittances remains constant after a certain number of cells in the longitudinal and horizontal plane that is, for the example set of beam parameters, 350 and 13 cells, respectively. Finally, the number of cells in the vertical plane can be calculated by $n_{y}=n_{x} /\left(\sigma_{x} / \sigma_{y}\right)$.

\section{Benchmarking of SIRE with the B-M IBS theoretical model}

SIRE has the advantage to accept any type of distribution as an input. If requested, it also gives as output the distribution at any stage of the tracking. In order to benchmark the code with the analytical formulation of B-M for the LHC, a Gaussian distribution was tracked for two sets of bunch parameters which are summarized in Table I for both the injection $(450 \mathrm{GeV})$ and collision energy $(6.5 \mathrm{TeV})$. The first case corresponds to the nominal BCMS [8,9] LHC beams, having a significantly lower transverse beam size with respect to the nominal production scheme. The second case corresponds to the HL-LHC $[10,51]$ parameters, for which the bunch population is very high. The input optics functions used for tracking correspond to the ones of the aforementioned reduced lattice.

\section{At the LHC injection energy (450 GeV)}

The evolution of the horizontal emittance (left), the vertical emittance (middle) and energy spread (right) after $1 \mathrm{~h}$ at injection energy $(450 \mathrm{GeV})$, where the IBS effect is dominant, are presented in Fig. 8 for the nominal BCMS case and in Fig. 9 for the HL-LHC parameters. The red and the blue lines correspond to the analytical calculations of

\footnotetext{
${ }^{1}$ Here it is assumed that the ratio of the transverse beam sizes is initially $\sigma_{x} / \sigma_{y}=1$.
}

the MAD-X [52] IBS routine (based on the B-M formalism) and to the SIRE results, respectively. Due to the fact that in SIRE the generation of the distribution is based on a random number generator, the tracking simulations were performed several times, resulting in the two standard deviation spread that are plotted in light blue. Table II summarizes the IBS growth of the transverse emittances and energy spread, for the nominal BCMS and HL-LHC parameters, as computed by the SIRE code and the B-M analytical formalism in MAD-X.

In the horizontal and longitudinal plane the IBS effect is dominant, while in the vertical plane, it is minor. Even though the SIRE simulation algorithm and the B-M analytical formalism make use of different approaches to calculate the IBS effect (SIRE uses the classical Rutherford cross section which is closer to the Piwinski formalism), they seem to agree very well during the $1 \mathrm{~h}$ time at injection energy. In the longitudinal plane, there is a small difference observed for longer time spans. Such differences can be explained by the fact that SIRE reshapes the beam distributions, in a self-consistent way, after each collisional process, while the B-M IBS formalism assumes Gaussian beam distributions throughout the calculation.

The variation of the initially Gaussian particle distributions within $1 \mathrm{~h}$ at injection energy is shown in logarithmic scale in Fig. 10 and Fig. 11 for the nominal BCMS and the HL-LHC case, respectively. The initial and final (after $1 \mathrm{~h}$ ) distributions in the horizontal (left), vertical (middle) and longitudinal (right) plane, are denoted by blue and red stars, respectively. They are fitted with the Gaussian (dashed line) and the $q$-Gaussian (solid line) functions. The q-Gaussian function allows to fit the full distribution, core and tail at the same time, providing also an rms size in a simple closed form, as shown in Eq. (A5), in Appendix A. The root mean square error (RMSE) is used as an error estimate between the fitted parameters of the distribution functions and the simulated ones. The fitting results of the initial and final distributions are presented in Table III for the nominal BCMS case and in Table IV for the HL-LHC case.

As was expected from the results shown in Figs. 8-9 concerning the IBS growth, the horizontal and longitudinal rms beam sizes get larger as time evolves, while the vertical one does not change. The vertical distributions remain Gaussian since $q \approx 1$. For both the nominal and the HLLHC case, the $q$ parameter of the horizontal and longitudinal distributions is decreased. As discussed in Sec. III, 

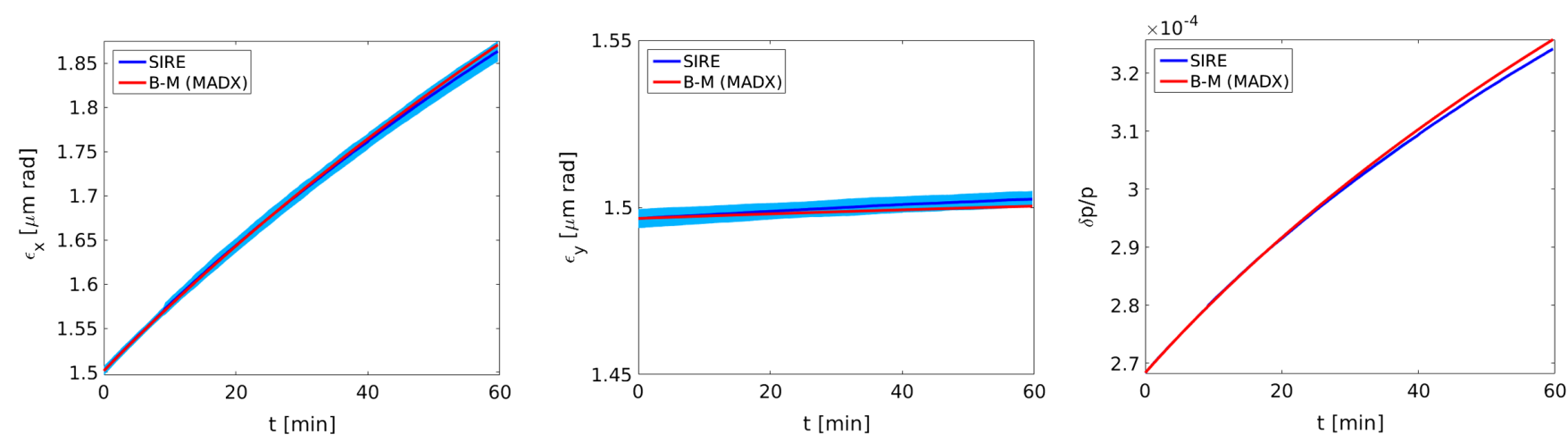

FIG. 8. The growth of the horizontal (left) and vertical (middle) emittance and energy spread (right) due to IBS, in a time period of $1 \mathrm{~h}$ at the injection energy of the LHC $(450 \mathrm{GeV})$ for the nominal parameters, as computed by the SIRE code (blue line) and the BjorkenMtingwa analytical formalism in MAD-X (red line).
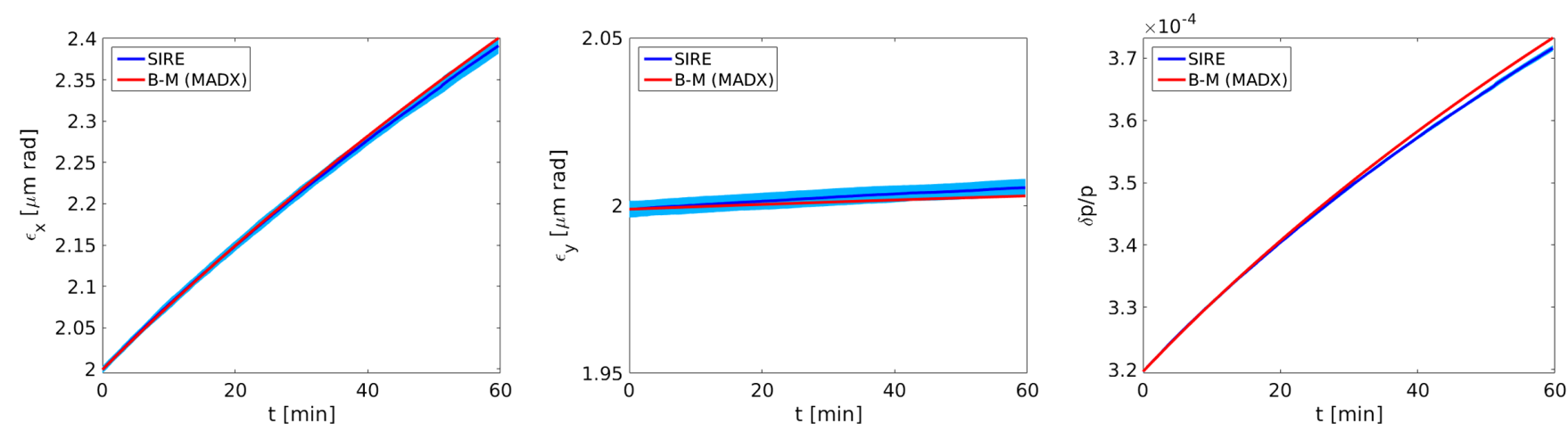

FIG. 9. The growth of the horizontal (left) and vertical (middle) emittance and energy spread (right) due to IBS, in a time period of $1 \mathrm{~h}$ at the injection energy of the LHC (450 GeV) for the HL-LHC parameters, as computed by the SIRE (blue line) and the BjorkenMtingwa analytical formalism in MAD-X (red line).

this can be explained by the fact that, due to IBS, the core of the distributions is blown up in such a way that it covers up the initially Gaussian tails of the input distributions, which remain less affected. In the longitudinal plane the decrease in $q$ is more significant for the HL-LHC case. This indicates that the stronger IBS is, the more the core is blown up. Since for a light tailed distribution $(q<1)$ the Gaussian fit overestimates the rms value, the resulted beam sizes are slightly larger than in the case of the $q$-Gaussian fit. Comparing the RMSE values of the two fitting functions for the final non-Gaussian bunch profiles shows that the $q$-Gaussian fit is better, in particular for the horizontal plane.

TABLE II. IBS growths of the transverse emittances and energy spread during $1 \mathrm{~h}$ at injection energy (450 GeV).

\begin{tabular}{lccccr}
\hline \hline & \multicolumn{2}{c}{ Nominal (BCMS) } & & \multicolumn{2}{c}{ HL-LHC } \\
\cline { 2 - 3 } \cline { 6 - 7 } IBS growths & MAD-X & SIRE & & MAD-X & SIRE \\
\hline$d \epsilon_{x} / \epsilon_{x 0}[\%]$ & 24.6 & 24.1 & & 20.1 & 19.6 \\
$d \epsilon_{y} / \epsilon_{y 0}[\%]$ & 0.2 & 0.4 & & 0.2 & 0.3 \\
$d \sigma_{l} / \sigma_{l 0}[\%]$ & 21.4 & 20.8 & & 16.8 & 16.2 \\
\hline \hline
\end{tabular}

\section{At the LHC collision energy (6.5 TeV)}

Since at collision energy IBS becomes weaker and synchrotron radiation starts playing an important role, it is the interplay between these effects that determines the evolution of the bunch characteristics. In this respect, for the benchmarking of the B-M IBS theoretical model with SIRE at collision energy, apart from the IBS, the radiation effects (synchrotron radiation and quantum excitation) are also taken into account. It should be mentioned that for the results presented in the following plots the intensity is assumed to be constant (which is actually true for the few noncolliding bunches, during physics fills).

Figure 12 shows the horizontal emittance (left), the vertical emittance (middle) and energy spread (right) evolution after $10 \mathrm{~h}$ at collision energy for the nominal BCMS case, while Fig. 13 shows the evolutions for the HL-LHC parameters. The red and the blue lines correspond to the analytical calculations of the MAD-X [52] IBS routine (based on the B-M formalism) and to the SIRE results, respectively. The two standard deviation error bars for the simulation results are plotted in light blue. Table $\mathrm{V}$ summarizes the variation of the transverse emittances and energy spread during $10 \mathrm{~h}$ at the collision energy of the 

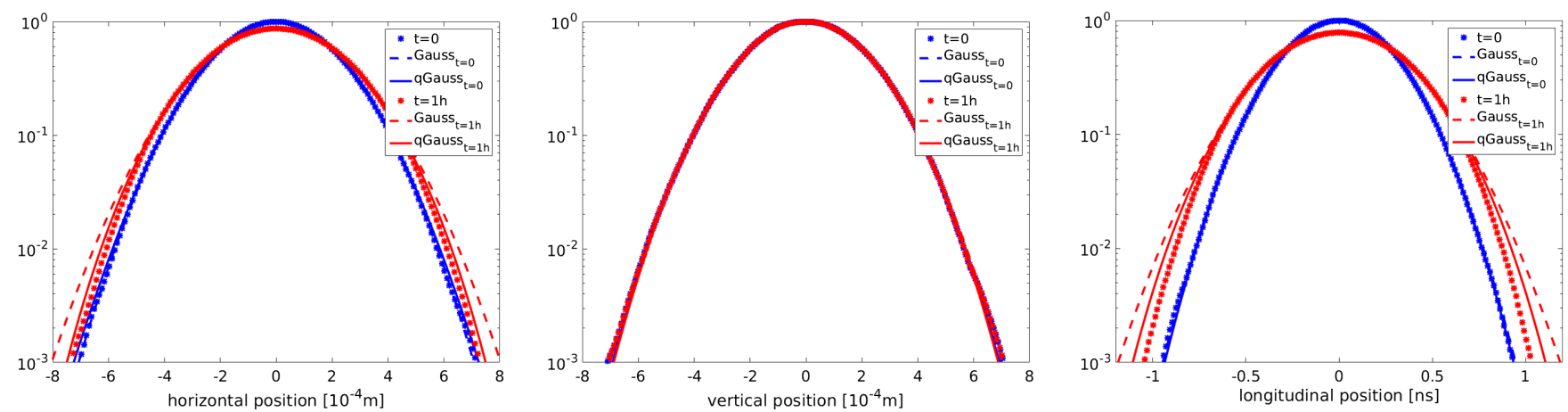

FIG. 10. The initial and final (after $1 \mathrm{~h}$ ) distributions in the horizontal (left), vertical (middle) and longitudinal (right) plane, for the nominal BCMS bunch parameters at injection energy $(450 \mathrm{GeV})$, are denoted by blue and red stars, respectively. They are fitted with the Gaussian (dashed line) and the q-Gaussian (solid line) functions.
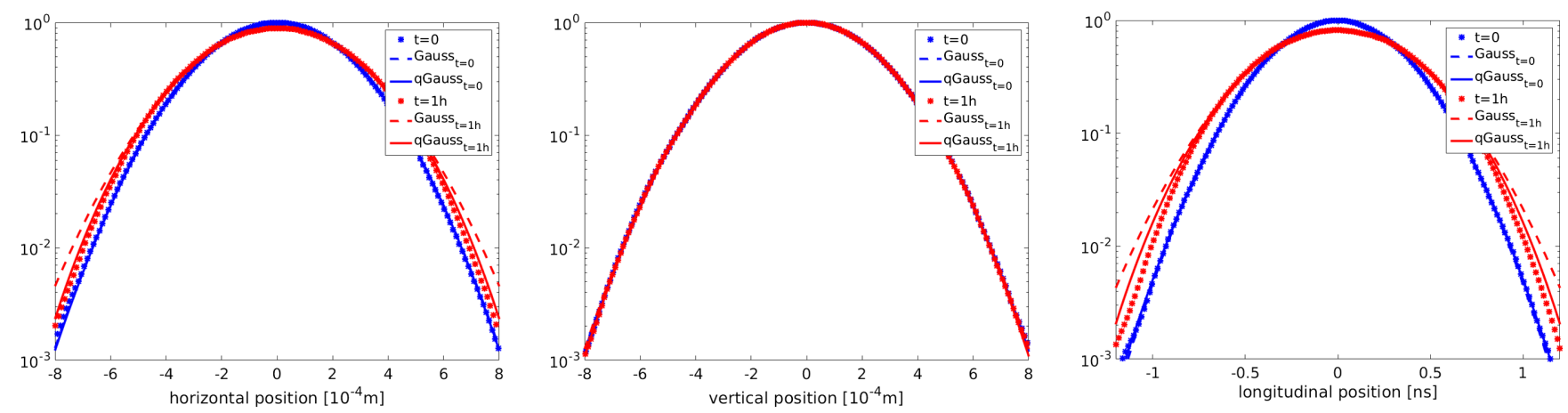

FIG. 11. The initial and final (after $1 \mathrm{~h}$ ) distributions in the horizontal (left), vertical (middle) and longitudinal (right) plane, for the HL-LHC bunch parameters at injection energy $(450 \mathrm{GeV})$, are denoted by blue and red stars, respectively. They are fitted with the Gaussian (dashed line) and the q-Gaussian (solid line) functions.

TABLE III. Initial and final (after $1 \mathrm{~h}$ ) fit results for the horizontal, vertical, and longitudinal bunch profiles shown in Fig. 10, for the nominal BCMS parameters case at injection energy (450 GeV).

\begin{tabular}{|c|c|c|c|c|c|c|c|}
\hline \multirow[b]{2}{*}{ Fit parameters } & & \multicolumn{2}{|c|}{ Horizontal distribution } & \multicolumn{2}{|c|}{ Vertical distribution } & \multicolumn{2}{|c|}{ Longitudinal distribution } \\
\hline & & Initial & Final & Initial & Final & Initial & Final \\
\hline$\overline{\text { Gaussian }}$ & $\begin{array}{c}\sigma_{\mathrm{rms}} \pm 10^{-3} \\
\mathrm{RMSE}\left[10^{-3}\right]\end{array}$ & $\begin{array}{c}0.19[\mathrm{~mm}] \\
1\end{array}$ & $\begin{array}{c}0.22[\mathrm{~mm}] \\
14\end{array}$ & $\begin{array}{c}0.19[\mathrm{~mm}] \\
1\end{array}$ & $\begin{array}{c}0.19[\mathrm{~mm}] \\
1\end{array}$ & $\begin{array}{c}0.25 \text { [ns] } \\
1\end{array}$ & $\begin{array}{c}0.33[\mathrm{~ns}] \\
10\end{array}$ \\
\hline q-Gaussian & $\begin{array}{c}\sigma_{\mathrm{rms}} \pm 10^{-3} \\
q \pm d q \\
\mathrm{RMSE}\left[10^{-3}\right]\end{array}$ & $\begin{array}{c}0.19[\mathrm{~mm}] \\
1.024 \pm 0.003 \\
1\end{array}$ & $\begin{array}{c}0.21[\mathrm{~mm}] \\
0.893 \pm 0.002 \\
1\end{array}$ & $\begin{array}{c}0.19[\mathrm{~mm}] \\
0.970 \pm 0.007 \\
1\end{array}$ & $\begin{array}{c}0.19[\mathrm{~mm}] \\
0.967 \pm 0.006 \\
1\end{array}$ & $\begin{array}{c}0.25[\mathrm{~ns}] \\
0.992 \pm 0.002 \\
1\end{array}$ & $\begin{array}{c}0.32[\mathrm{~ns}] \\
0.941 \pm 0.001 \\
6\end{array}$ \\
\hline
\end{tabular}

TABLE IV. Initial and final (after $1 \mathrm{~h}$ ) fitting results for the horizontal, vertical, and longitudinal bunch profiles shown in Fig. 11, for the HL-LHC parameters case at injection energy $(450 \mathrm{GeV})$.

\begin{tabular}{|c|c|c|c|c|c|c|c|}
\hline \multirow[b]{2}{*}{ Fit parameters } & & \multicolumn{2}{|c|}{ Horizontal distribution } & \multicolumn{2}{|c|}{ Vertical distribution } & \multicolumn{2}{|c|}{ Longitudinal distribution } \\
\hline & & Initial & Final & Initial & Final & Initial & Final \\
\hline Gaussian & $\begin{array}{c}\sigma_{\mathrm{rms}} \pm 10^{-3} \\
\mathrm{RMSE}\left[10^{-3}\right]\end{array}$ & $\begin{array}{c}0.22[\mathrm{~mm}] \\
1\end{array}$ & $\begin{array}{c}0.25[\mathrm{~mm}] \\
14\end{array}$ & $\begin{array}{c}0.22[\mathrm{~mm}] \\
1\end{array}$ & $\begin{array}{c}0.22[\mathrm{~mm}] \\
1\end{array}$ & $\begin{array}{c}0.30[\mathrm{~ns}] \\
3\end{array}$ & $\begin{array}{c}0.37[\mathrm{~ns}] \\
13\end{array}$ \\
\hline q-Gaussian & $\begin{array}{c}\sigma_{\mathrm{rms}} \pm 10^{-3} \\
q \pm d q \\
\mathrm{RMSE}\left[10^{-3}\right]\end{array}$ & $\begin{array}{c}0.22[\mathrm{~mm}] \\
0.992 \pm 0.003 \\
1\end{array}$ & $\begin{array}{c}0.24[\mathrm{~mm}] \\
0.891 \pm 0.004 \\
1\end{array}$ & $\begin{array}{c}0.22[\mathrm{~mm}] \\
0.995 \pm 0.003 \\
1\end{array}$ & $\begin{array}{c}0.22[\mathrm{~mm}] \\
0.987 \pm 0.003 \\
1\end{array}$ & $\begin{array}{c}0.30[\mathrm{~ns}] \\
1.019 \pm 0.005 \\
3\end{array}$ & $\begin{array}{c}0.36[\mathrm{~ns}] \\
0.885 \pm 0.001 \\
4\end{array}$ \\
\hline
\end{tabular}



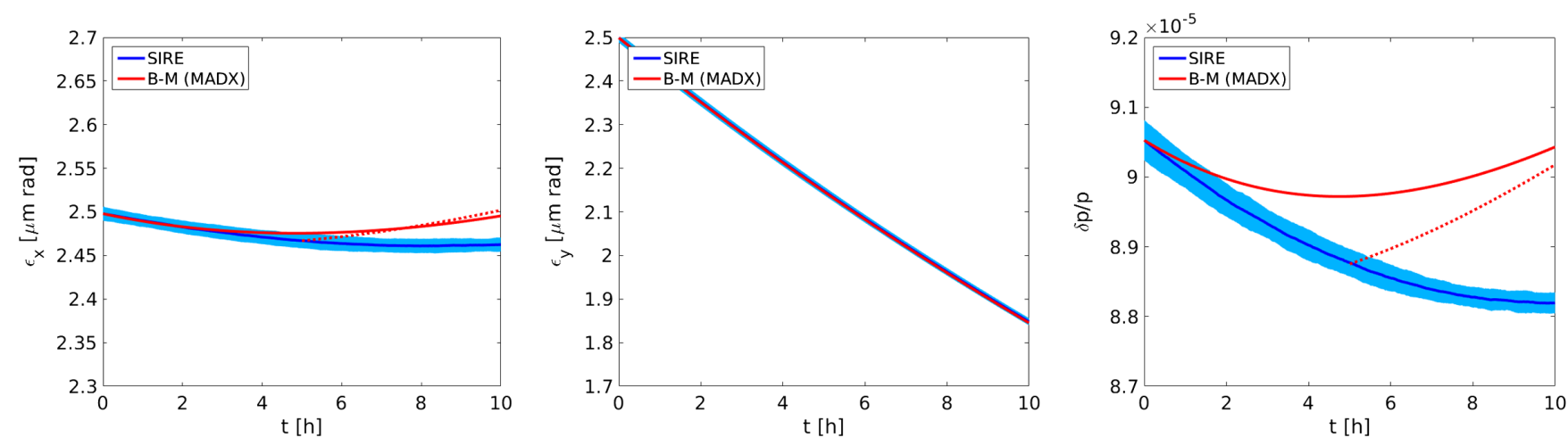

FIG. 12. The evolution of the horizontal (left) and vertical (middle) emittance and energy spread (right) due to IBS and radiation effects, in a time period of $10 \mathrm{~h}$ at the collision energy of the LHC (6.5 TeV) for the nominal BCMS parameters, as computed by the SIRE code (blue line) and the Bjorken-Mtingwa analytical formalism in MAD-X (red line).
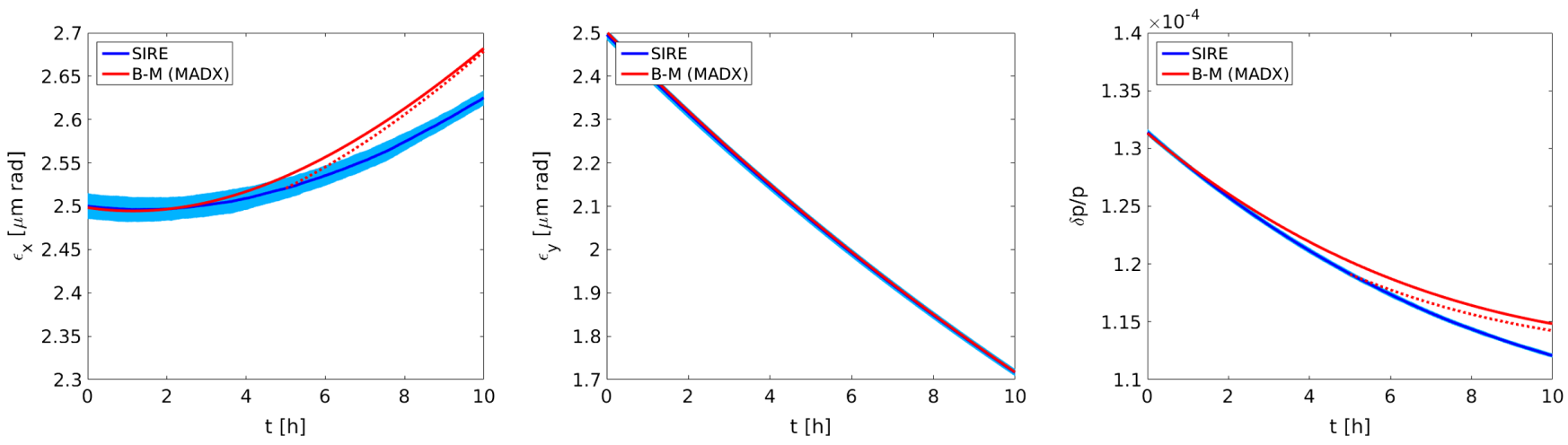

FIG. 13. The evolution of the horizontal (left) and vertical (middle) emittance and energy spread (right) due to IBS and radiation effects, in a time period of $10 \mathrm{~h}$ at the collision energy of the LHC (7 TeV) for the HL-LHC parameters, as computed by the SIRE (blue line) and the Bjorken-Mtingwa analytical formalism in MAD-X (red line).

LHC, for the nominal BCMS and HL-LHC parameters, as computed by the SIRE code and the B-M analytical formalism in MAD-X.

After a few hours at collisions, the B-M analytical formalism and the simulations start differentiating. In order to understand whether these differences are explained by the fact that SIRE reshapes the beam distributions after each collisional process and the B-M IBS formalism assumes always Gaussian beam distributions, the bunch parameters given by SIRE at $5 \mathrm{~h}$ are used as input for the IBS and synchrotron radiation calculations in MAD-X (Gaussian bunches). The red dotted lines in Fig. 12 and

TABLE V. Variation of the transverse emittances and energy spread during $10 \mathrm{~h}$ at FT energy.

\begin{tabular}{lcrrrr}
\hline \hline & \multicolumn{2}{c}{ Nominal (BCMS) } & & \multicolumn{2}{c}{ HL-LHC } \\
\cline { 2 - 3 } \cline { 6 - 6 } IBS growths & MAD-X & SIRE & & MAD-X & SIRE \\
\hline$d \epsilon_{x} / \epsilon_{x 0}[\%]$ & -0.1 & -1.4 & & 7.4 & 5.0 \\
$d \epsilon_{y} / \epsilon_{y 0}[\%]$ & -26.2 & -26.1 & & -31.4 & -31.2 \\
$d \sigma_{l} / \sigma_{l 0}[\%]$ & -0.1 & -2.6 & & -12.6 & -14.7 \\
\hline \hline
\end{tabular}

Fig. 13 represent the results of these tests. Even if giving as input to MAD-X exactly the same bunch parameters as in SIRE, there is clear divergence of the MAD-X results (red dotted lines) with SIRE right after the $5 \mathrm{~h}$ at collisions. This divergence is much larger than the one observed during the first hours at collisions. After $5 \mathrm{~h}$ at collision energy, the beam in SIRE has been reshaped enough so that IBS and radiation processes act differently as compared to Gaussian MAD-X distributions. Consequently, the differences observed between the B-M analytical formalism and the simulations are expected because MAD-X assumes always Gaussian distribution, in contrast to SIRE that takes into account the variation of the bunch shape throughout the calculation.

Due to the fact that the IBS effect is minor in the vertical plane, the strong synchrotron radiation damping mechanism leads to a clear reduction of the vertical emittance. However, the variation of the horizontal emittance and energy spread is determined by the interplay of IBS growth with synchrotron radiation damping. For the nominal BCMS parameters, these variations are very small after $10 \mathrm{~h}$ at collision energy (Table V). For the HL-LHC case, 

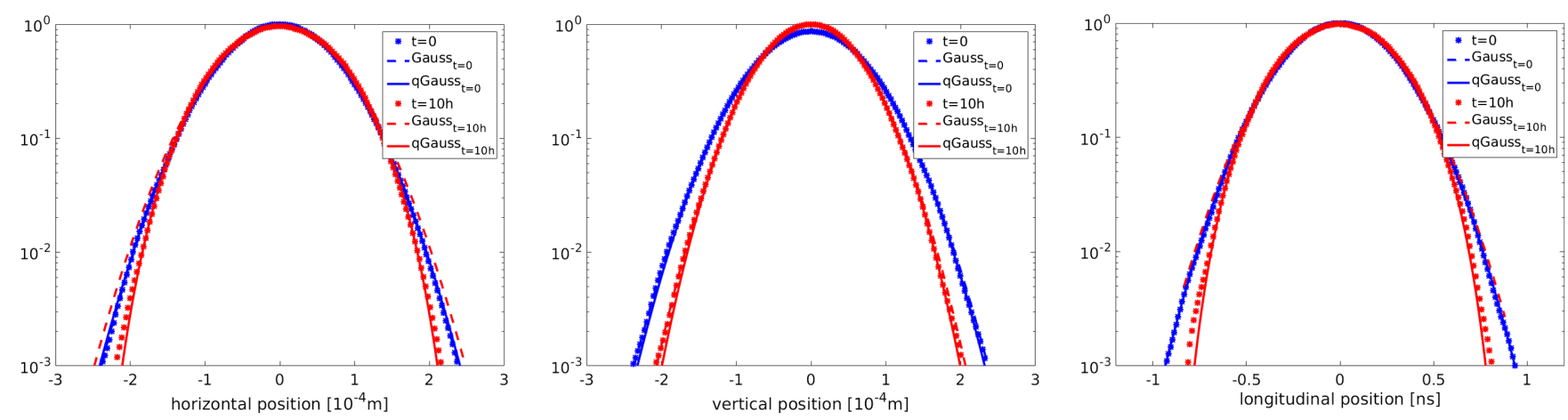

FIG. 14. The initial and final (after $10 \mathrm{~h}$ ) distributions in the horizontal (left), vertical (middle), and longitudinal (right) plane, for the nominal BCMS bunch parameters at collision energy $(6.5 \mathrm{TeV})$, are denoted by blue and red stars, respectively. They are fitted with the Gaussian (dashed line) and the q-Gaussian (solid line) functions.
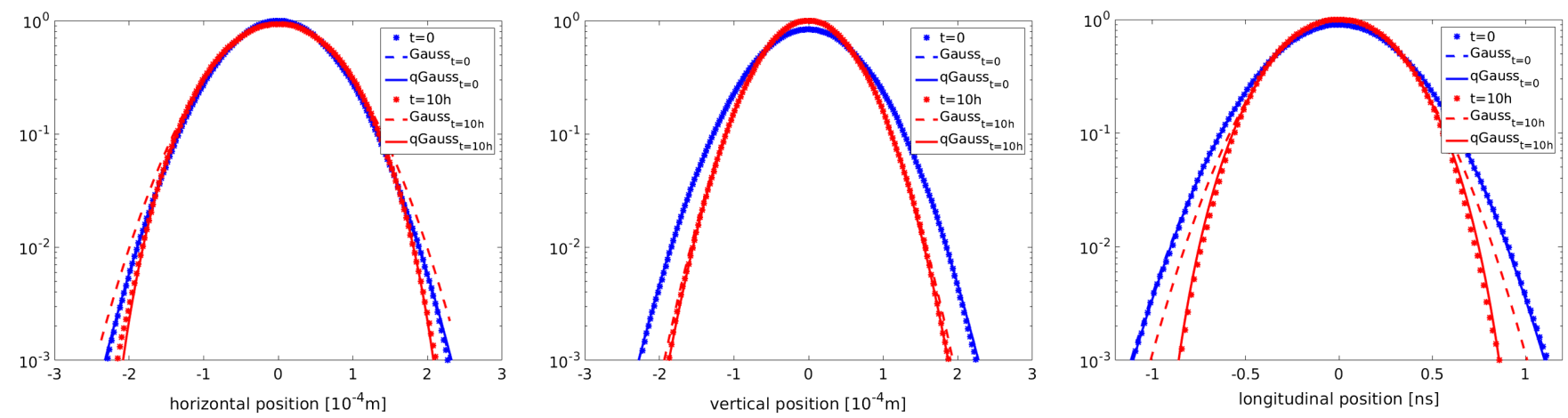

FIG. 15. The initial and final (after $10 \mathrm{~h}$ ) distributions in the horizontal (left), vertical (middle), and longitudinal (right) plane, for the HL-LHC bunch parameters at collision energy ( $7 \mathrm{TeV}$ ), are denoted by blue and red stars, respectively. They are fitted with the Gaussian (dashed line) and the q-Gaussian (solid line) functions.

having the same initial horizontal emittance but the double bunch population compared to the nominal BCMS parameters (Table I), the IBS effect prevails over synchrotron radiation in the horizontal plane after almost $3 \mathrm{~h}$ [Fig. 13 (left)]. As can be seen in Fig. 13 (right) this in not the case for the longitudinal plane, for which the initial bunch length of $1.2 \mathrm{~ns}$ compared to the $1 \mathrm{~ns}$ in the nominal case, renders IBS weaker than synchrotron radiation and, results in the decrease of the energy spread.

The evolution of the initially Gaussian (in all planes) particle distributions within $10 \mathrm{~h}$ at collision energy is shown in logarithmic scale in Fig. 14 and Fig. 15 for the nominal BCMS and the HL-LHC case, respectively. The initial and final (after $10 \mathrm{~h}$ ) distributions in the horizontal (left), vertical (middle) and longitudinal (right) plane, are denoted by blue and red stars, respectively. They are fitted with the Gaussian (dashed line) and the $q$-Gaussian (solid line) functions. The fitting results of the initial and final distributions are presented in Table VI for the nominal BCMS case and in Table VII for the HL-LHC case. The RMSE values of the two fitting functions show that when the final bunch profiles are strongly non-Gaussian, the q-Gaussian fitting results should be considered. In this respect, the evolution of the particle distributions in all

TABLE VI. Initial and final (after $10 \mathrm{~h}$ ) fit results for the horizontal, vertical, and longitudinal bunch profiles shown in Fig. 14, for the nominal BCMS parameters case at collision energy $(6.5 \mathrm{TeV})$.

\begin{tabular}{|c|c|c|c|c|c|c|c|}
\hline \multirow[b]{2}{*}{ Fit parameters } & & \multicolumn{2}{|c|}{ Horizontal distribution } & \multicolumn{2}{|c|}{ Vertical distribution } & \multicolumn{2}{|c|}{ Longitudinal distribution } \\
\hline & & Initial & Final & Initial & Final & Initial & Final \\
\hline Gaussian & $\begin{array}{c}\sigma_{\mathrm{rms}} \pm 10^{-4} \\
\mathrm{RMSE}\left[10^{-3}\right]\end{array}$ & $\begin{array}{c}0.064[\mathrm{~mm}] \\
1\end{array}$ & $\begin{array}{c}0.067[\mathrm{~mm}] \\
25\end{array}$ & $\begin{array}{c}0.064[\mathrm{~mm}] \\
1\end{array}$ & $\begin{array}{c}0.056[\mathrm{~mm}] \\
1\end{array}$ & $\begin{array}{c}0.25[\mathrm{~ns}] \\
1\end{array}$ & $\begin{array}{c}0.26[\mathrm{~ns}] \\
30\end{array}$ \\
\hline q-Gaussian & $\begin{array}{c}\sigma_{\mathrm{rms}} \pm 10^{-4} \\
q \pm d q \\
\mathrm{RMSE}\left[10^{-3}\right]\end{array}$ & $\begin{array}{c}0.064[\mathrm{~mm}] \\
1.004 \pm 0.003 \\
1\end{array}$ & $\begin{array}{c}0.064[\mathrm{~mm}] \\
0.856 \pm 0.005 \\
1\end{array}$ & $\begin{array}{c}0.064[\mathrm{~mm}] \\
0.982 \pm 0.004 \\
1\end{array}$ & $\begin{array}{c}0.055[\mathrm{~mm}] \\
0.971 \pm 0.004 \\
1\end{array}$ & $\begin{array}{c}0.25[\mathrm{~ns}] \\
1.007 \pm 0.004 \\
1\end{array}$ & $\begin{array}{c}0.24[\mathrm{~ns}] \\
0.830 \pm 0.006 \\
1\end{array}$ \\
\hline
\end{tabular}


TABLE VII. Initial and final (after $10 \mathrm{~h}$ ) fitting results for the horizontal, vertical, and longitudinal bunch profiles shown in Fig. 15, for the HL-LHC parameters case at collision energy (7 TeV).

\begin{tabular}{|c|c|c|c|c|c|c|c|}
\hline \multirow[b]{2}{*}{ Fit parameters } & & \multicolumn{2}{|c|}{ Horizontal distribution } & \multicolumn{2}{|c|}{ Vertical distribution } & \multicolumn{2}{|c|}{ Longitudinal distribution } \\
\hline & & Initial & Final & Initial & Final & Initial & Final \\
\hline Gaussian & $\begin{array}{c}\sigma_{\mathrm{rms}} \pm 10^{-4} \\
\mathrm{RMSE}\left[10^{-3}\right]\end{array}$ & $\begin{array}{c}0.062[\mathrm{~mm}] \\
1\end{array}$ & $\begin{array}{c}0.067[\mathrm{~mm}] \\
27\end{array}$ & $\begin{array}{c}0.062[\mathrm{~mm}] \\
2\end{array}$ & $\begin{array}{c}0.052[\mathrm{~mm}] \\
2\end{array}$ & $\begin{array}{c}0.30[\mathrm{~ns}] \\
2\end{array}$ & $\begin{array}{c}0.28[\mathrm{~ns}] \\
17\end{array}$ \\
\hline q-Gaussian & $\begin{array}{c}\sigma_{\mathrm{rms}} \pm 10^{-4} \\
q \pm d q \\
\mathrm{RMSE}\left[10^{-3}\right]\end{array}$ & $\begin{array}{c}0.062[\mathrm{~mm}] \\
1.005 \pm 0.004 \\
1\end{array}$ & $\begin{array}{c}0.063[\mathrm{~mm}] \\
0.852 \pm 0.004 \\
1\end{array}$ & $\begin{array}{c}0.062[\mathrm{~mm}] \\
0.991 \pm 0.005 \\
1\end{array}$ & $\begin{array}{c}0.052[\mathrm{~mm}] \\
0.977 \pm 0.005 \\
1\end{array}$ & $\begin{array}{c}0.30[\mathrm{~ns}] \\
0.990 \pm 0.003 \\
1\end{array}$ & $\begin{array}{c}0.27[\mathrm{~ns}] \\
0.825 \pm 0.001 \\
1\end{array}$ \\
\hline
\end{tabular}

planes for the nominal BCMS and HL-LHC cases is discussed based on the $q$-Gaussian results.

The horizontal beam sizes do not change after $10 \mathrm{~h}$ at collision energy because the blow up caused by IBS is balanced out by the synchrotron radiation damping. However, there is a transformation of the horizontal distributions' shape for which the tails become less populated $(q<1)$. In the longitudinal plane both the beam size and the $q$ parameter are reduced, meaning that synchrotron radiation prevails over IBS and the core is blown up due to IBS giving underpopulated tails. In the vertical plane, the dominant synchrotron radiation damping results in a smaller beam size without changing much the formation of the tails, so the distribution remains Gaussian.

\section{BUNCH PROFILE MEASUREMENTS IN THE LHC}

The transverse diagnostic instruments for measuring the bunch profiles in the LHC are the betatron matching monitor [53], the beam gas ionization (BGI) monitor [54], the beam gas vertex (BGV) monitor, the beam wire scanners (WS) [55], and the beam synchrotron light monitor (BSRT) [56]. Compatibly with high intensity and high energy operation, the BSRT is the only instrument offering noninvasive, continuous, and bunch-by-bunch measurements of the LHC beams. The BSRT is calibrated with respect to the WS during dedicated low beam intensity runs. $^{2}$ The LHC is equipped with two synchrotron radiation monitors (one per beam) used to characterize the transverse and longitudinal beam distributions. Due to the significant diffraction patterns at the tails of the BSRT transverse distributions there is no clear picture about the shape of the tails and so, they are often assumed to be Gaussian.

A parameter that is generally used to measure the longitudinal emittance in circular accelerators is the bunch length. The bunch length is given by the projection of the distribution function on the phase axis, which is known as

\footnotetext{
${ }^{2}$ The WS can measure the emittance throughout the full LHC machine cycle including the energy ramp, provided that the total intensity in the machine is limited to 240 nominal bunches at $450 \mathrm{GeV}$ and 12 nominal bunches at $6.5 \mathrm{TeV}$.
}

the bunch profile or line density. It is operationally measured by the LHC beam quality monitor (BQM) [57] which uses a wall current monitor pick-up (WCM) [58] to acquire the longitudinal profiles. Additionally, the longitudinal synchrotron radiation monitor (BSRL) [59], which uses the same synchrotron light source as the BSRT, continuously measures the longitudinal distribution of charges in the beams. The scopes connected to the WCM pick-ups can acquire longitudinal bunch profiles of both beams during a full LHC cycle.

There is a filtering procedure to remove background noise in all the measured profiles. There is also an automatic gain which deals with the dynamic range of the monitors in order to avoid mainly saturation, but in the case of the LHC, all the measurements are done within very similar beam parameters and thereby, monitor settings. In addition, the measurements at the LHC injection energy $(450 \mathrm{GeV})$ are very well calibrated with other emittance monitors, the ones using synchrotron light against traditional wire-scanners. In order to remove any further "noise" effect, in particular in the tails of the distributions, such as optical diffraction, fast Fourier transform algorithms for filtering and 3 sigma cuts were also applied to the data [60].

\section{A. Comparison between experimental data, the SIRE, and the B-M analytical formalism, at the LHC collision energy $(6.5 \mathrm{TeV})$}

The longitudinal bunch manipulations performed during the ramp to avoid instabilities due to the loss of Landau damping [16], produce bunches that arrive at collision energy with a clearly non-Gaussian longitudinal shape. In addition, the transfer functions of the pickups and cables were measured and are used for deconvolution [61], resulting in some cases in tails which are asymmetric or have ripples. By assuming that these profiles are Gaussian may lead in underestimating or overestimating the actual bunch length. For the studies presented in this paper, these profiles are fitted using the $q$-Gaussian function. An example showing the evolution of the $q$ parameter for the longitudinal profile of a bunch train during $11.5 \mathrm{~h}$ at collisions $(6.5 \mathrm{TeV})$ in the LHC is presented in Fig. 16. It is clear that with such $q$ parameter values, corresponding to 


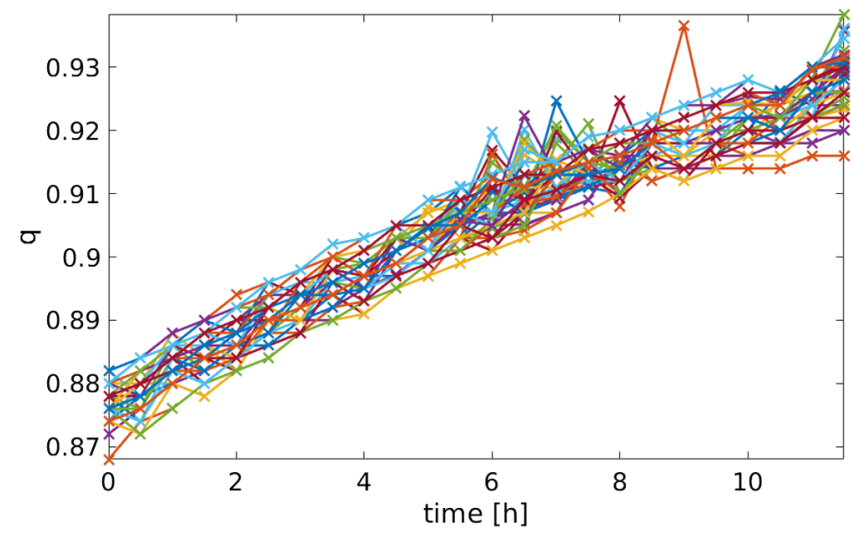

FIG. 16. The evolution of the $q$ parameter during $11.5 \mathrm{~h}$ at collisions $(6.5 \mathrm{TeV})$, for a train of bunches in the longitudinal plane.

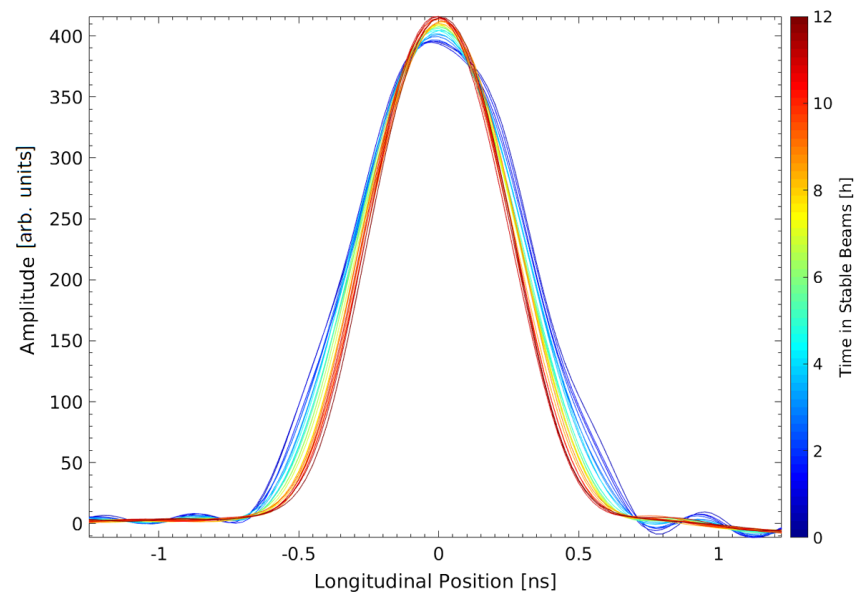

FIG. 17. The evolution of a longitudinal bunch profile during $11.5 \mathrm{~h}$ at collisions $(6.5 \mathrm{TeV})$.

non-Gaussian tails, the rms beam size cannot be accurately estimated by using the Gaussian function. The increase of the $q$ parameter means that the longitudinal distributions

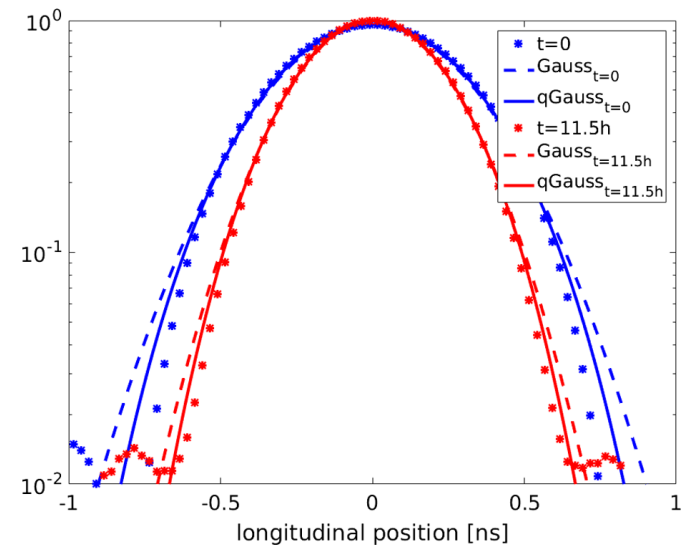

with the underpopulated tails $(q<1)$ at the start of collisions, become more Gaussian $(q \rightarrow 1)$ as time evolves. This is a general statement that can be made for the longitudinal distribution observed at the collision energy of the LHC.

Figure 16 shows measured longitudinal profiles for colliding bunches where the evolution (the reduction) of the bunch intensity is present. Since IBS is getting weaker and synchrotron radiation dominates, for these bunches that arrive at the start of collisions with light tails, the $q$ value increases and the rms size decreases with time. For the simulation results shown in Table VI the trend is different because they are conducted with a constant bunch intensity. These simulations were undertaken for evaluating the combined effect of IBS and synchrotron radiation to the beam distribution, without any other mechanism present (beam-beam, etc.), starting from a purely Gaussian one. They indeed show that the $q$ value decreases with time in all planes. Specifically, in the longitudinal plane, the simulations show that $q$ value decreases by approximately $1.7 \% / h$. In fact, this trend agrees with what is observed in the LHC for the non-colliding bunches (having constant intensity) in the longitudinal plane, where it is measured that there is a $1.5 \% / h$ reduction of the $q$ value.

The evolution of the longitudinal particle distribution of a single bunch that is picked out of the train of bunches is shown in Fig. 17 for the time period of $11.5 \mathrm{~h}$. The initial bunch profile (plotted in blue) is fitted with the Gaussian and the q-Gaussian functions that give different rms beam sizes because of the dependence of the standard deviation on the $q$ parameter (Eq. (A5). The fitting results are used to generate a Gaussian and a q-Gaussian distribution to be tracked in SIRE in order to compare the experimental observations with the results of the code.

In Fig. 18, the initial (at the start of collisions) and the final (after $11.5 \mathrm{~h}$ ) longitudinal bunch profiles, as observed in the LHC (left) and as calculated by SIRE (right) for an initially $q$-Gaussian simulated profile, are denoted by blue

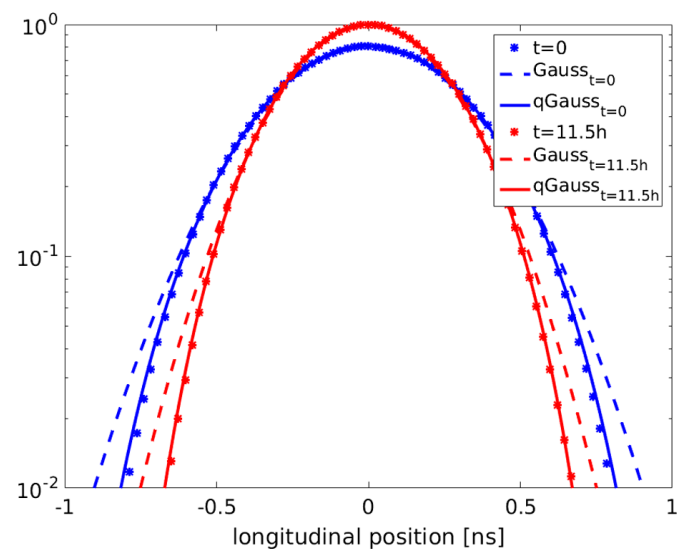

FIG. 18. The initial (at the start of collisions) and the final (after $11.5 \mathrm{~h}$ ) longitudinal bunch profiles as observed in the LHC (left) and as calculated by the SIRE (right), in logarithmic scale, are denoted by blue and red stars, respectively. They are fitted with the Gaussian (dashed line) and the $q$-Gaussian (solid line) functions. 
TABLE VIII. Fitting results for the initial (at the start of collisions) and the final (after $11.5 \mathrm{~h}$ ) longitudinal bunch distribution shown in Fig. 18, as was observed in the LHC and as was calculated by the SIRE code.

\begin{tabular}{|c|c|c|c|c|c|}
\hline \multirow[b]{2}{*}{ Fit parameters } & & \multicolumn{2}{|c|}{ Initial $(t=0)$} & \multicolumn{2}{|c|}{ Final $(t=11.5 \mathrm{~h})$} \\
\hline & & DATA & SIRE & DATA & SIRE \\
\hline Gaussian & $\begin{array}{c}\sigma_{\mathrm{rms}} \pm d \sigma_{\mathrm{rms}}[\mathrm{ns}] \\
\mathrm{RMSE}\left[10^{-3}\right]\end{array}$ & $\begin{array}{c}0.299 \pm 0.003 \\
22\end{array}$ & $\begin{array}{c}0.297 \pm 0.002 \\
19\end{array}$ & $\begin{array}{c}0.233 \pm 0.002 \\
18\end{array}$ & $\begin{array}{c}0.237 \pm 0.002 \\
20\end{array}$ \\
\hline q-Gaussian & $\begin{array}{c}\sigma_{\mathrm{rms}} \pm d \sigma_{\mathrm{rms}}[\mathrm{ns}] \\
q \pm d q \\
\mathrm{RMSE}\left[10^{-3}\right]\end{array}$ & $\begin{array}{c}0.286 \pm 0.004 \\
0.88 \pm 0.03 \\
10\end{array}$ & $\begin{array}{c}0.290 \pm 0.001 \\
0.85 \pm 0.01 \\
3\end{array}$ & $\begin{array}{c}0.227 \pm 0.002 \\
0.93 \pm 0.03 \\
10\end{array}$ & $\begin{array}{c}0.235 \pm 0.001 \\
0.86 \pm 0.01 \\
4\end{array}$ \\
\hline
\end{tabular}

and red stars, respectively. They are plotted in logarithmic scale and they are fitted with the Gaussian (dashed line) and the $q$-Gaussian (solid line) functions. The reduction of the bunch population with time due to burn-off and the extra (on top of IBS) transverse emittance blow-up observed in the machine, are taken into account for the simulation. The transverse distributions are assumed to be Gaussian, since at collisions the shape of their tails is not clear due to diffraction. The fitting results are presented in Table VIII. Even if there seems to be no change at the tails of the simulated distribution, in reality the profiles become more Gaussian. Within $11.5 \mathrm{~h}$ at collisions, the rms beam size of the measured bunch profile and of the corresponding tracked distribution is reduced by $21 \%$ and by $19 \%$, respectively. This shows a very good agreement between the experimental data and the simulations performed with SIRE.

Figure 19 shows in logarithmic scale the initial (blue stars) and the final (red stars) horizontal (left) and vertical (right) bunch profiles as calculated by SIRE, fitted with the Gaussian (dashed line) and the $q$-Gaussian (solid line) functions. As can be seen in Table IX, the simulations showed no change in the transverse beam sizes and that is because the extra (on top of IBS) transverse emittance blow-up is included. The effect of IBS together with the extra blow-up assumed, widens the core of the horizontal bunch in such a way that the $q$ parameter is decreased by around $10 \%$ within these $11.5 \mathrm{~h}$. Since IBS is negligible in the vertical plane, the fact that the vertical bunch profile remains Gaussian indicates that the interplay between the synchrotron radiation damping and the extra blow-up do not change the tails of the distribution.

The $4 \sigma$-bunch length evolution when assuming Gaussian (left) and $q$-Gaussian (right) initial distributions is shown in Fig. 20. The blue line corresponds to the SIRE calculations and the red line to the results given by the IBS module of MAD-X [24] which is based on the analytical formulation of B-M and always assumes Gaussian distributions. The bunch length evolution, together with the two standard deviation error-bars, when fitting the data with the Gaussian and the q-Gaussian functions is represented by a black and a grey line, respectively. The bunch length values differ for the two distribution functions used due to the fact that, for a light tailed distribution the rms value is overestimated by fitting a Gaussian. When assuming a Gaussian distribution, the bunch length evolution calculated by the code gets closer to the measured data. For the $q$-Gaussian case the agreement between data and simulations is excellent. This is a remarkable result, taking into account the fact that no assumptions are being made in the
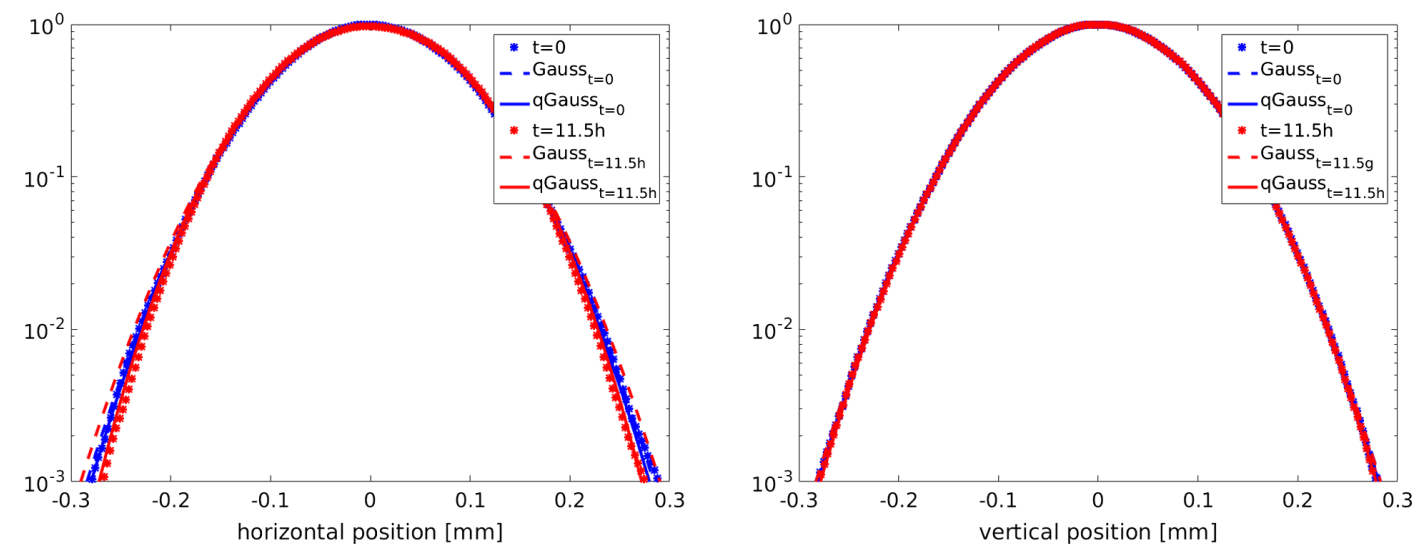

FIG. 19. The initial (at the start of collisions) and the final (after $11.5 \mathrm{~h}$ ) horizontal (left) and vertical (right) bunch profiles as calculated by the SIRE, in logarithmic scale, are denoted by blue and red stars, respectively. They are fitted with the Gaussian (dashed line) and the q-Gaussian (solid line) functions. 
TABLE IX. Fitting results for the initial (at the start of collisions) and the final (after $11.5 \mathrm{~h}$ ) transverse bunch distributions shown in Fig. 19, as was calculated using the SIRE code.

\begin{tabular}{lccccccc}
\hline \hline \multirow{2}{*}{ Fit parameters } & & \multicolumn{2}{c}{ Horizontal distribution } & & \multicolumn{2}{c}{ Vertical distribution } \\
\cline { 3 - 4 } & & Initial & Final & & Initial & Final \\
\hline q-Gaussian & $\sigma_{\text {rms }} \pm 10^{-4}[\mathrm{~mm}]$ & 0.076 & 0.076 & & 0.076 & 0.076 \\
& $q \pm d q$ & $0.990 \pm 0.004$ & $0.893 \pm 0.005$ & & $0.992 \pm 0.003$ & $0.983 \pm 0.003$ \\
& RMSE $\left[10^{-3}\right]$ & 3 & 3 & & 3 & 3 \\
\hline \hline
\end{tabular}

simulations apart from identical initial conditions with respect to the experimental ones. In agreement with the results presented in the previous section, the divergence between the SIRE and the MAD-X for longer time spans is something to be expected since the distribution shape in SIRE is updated, while in MAD-X it remains Gaussian.

In Sec. II the impact of non-Gaussian beam distributions on the luminosity was discussed. In the case of the LHC, we can only be confident about the impact of the longitudinal distributions, as the transverse bunch profile measurements are limited by diffraction patterns in the far tail regime. As an example, the luminosity discrepancy is calculated for the case of a Gaussian $(q=1)$ and a light tailed q-Gaussian with $q=0.88$ (based on longitudinal bunch profiles observations), which correspond to $1.20 \mathrm{~ns}$ and 1.14 ns bunch length (see Fig. 20), respectively. Keeping the rest of the beam parameters fixed, the Gaussian distribution gives a luminosity that is $3.5 \%$ lower compared to one in the case of the light tailed distribution. In fact, this is a systematic error beyond the luminosity precision target of $1 \%$ required by the experiments and it was communicated to them in order to be employed for a more accurate luminosity estimate.

At collisions, the divergence between the luminosity model [1] and the measured luminosity by the experiments becomes more pronounced as time evolves [3]. Actually, the predicted luminosity by the model is always larger compared to the measured one by the end of collisions.

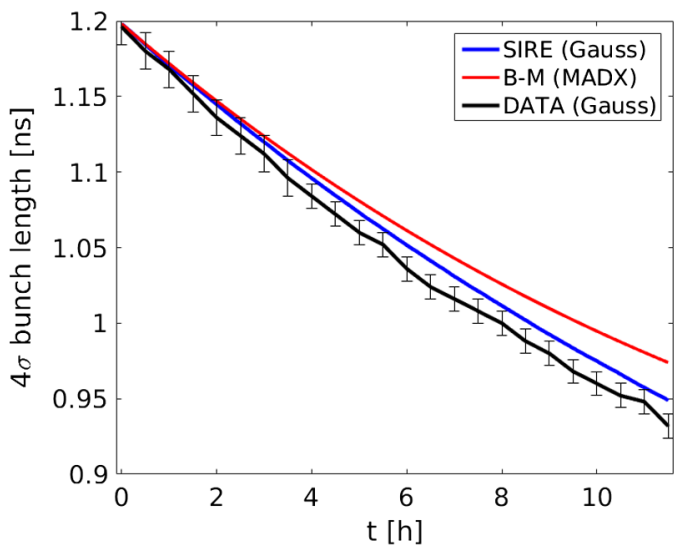

As calculated by SIRE, the weight of the horizontal bunch profile tails is decreased in time (see Table IX) and as explained in Sec. II, for lighter tails and a constant beam size, the luminosity is expected to become lower. It is then clear that by taking into account the luminosity change due to the variation of the transverse distribution tails, the model predictions can be significantly improved.

\section{SUMMARY AND OUTLOOK}

In the LHC, the interplay between a series of effects can lead to distributions with non-Gaussian tails. Since the rms value of a distribution can be underestimated or overestimated by using a simple Gaussian function, the use of appropriate fitting functions to accurately estimate the beam size and the behavior of the tails is necessary. The impact of non-Gaussian distribution shapes on the estimated luminosity is discussed. One of the next steps is to improve the luminosity model, that is currently based on Gaussian distributions, by taking into account the actual shape of the bunch profiles. In this way, it is possible to get more accurate luminosity predictions. Already, for the operational scenario of the high luminosity LHC upgrade [51], a nonGaussian bunch length estimation is being considered.

The way IBS and radiation effects act depends on the shape of the bunch profiles. Aiming to quantify the impact of the distribution's shape on the emittance evolution, a multiparticle tracking code called SIRE, is used.

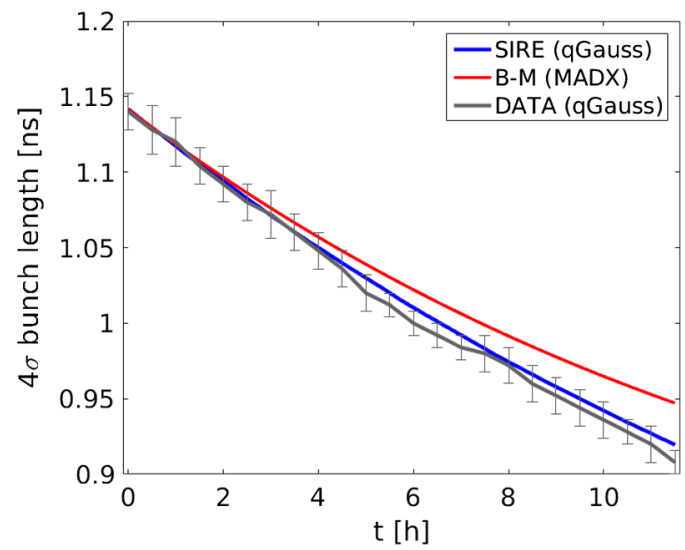

FIG. 20. The bunch length ( $4 \sigma$ ) evolution during several hours in collisions, as computed by the SIRE code (blue), the B-M analytical formalism (red) and as measured by the longitudinal profile monitors when assuming a Gaussian (left) and a q-Gaussian (right) distribution. 
The benchmarking of the B-M analytical formalism with SIRE showed a very good agreement for the first couple of hours at the injection $(450 \mathrm{GeV})$ and collision $(6.5 \mathrm{TeV})$ energies of the LHC, even if they make use of different approaches to calculate the IBS effect. The differences observed for longer time-spans are expected, since in SIRE the particle distributions are updated, while MAD-X always assumes Gaussian distributions. The results obtained from the simulations encourage the idea of using the code for tracking distributions coming from experimental data, in order to study the impact of the distributions shape on the evolution of the bunch characteristics. Specifically, the tracking is performed using the observed longitudinal beam distributions for which the measurements at the LHC collision energy are accurate enough, assuming that the transverse profiles are Gaussian. After the comparison with experimental data, the fact that SIRE takes into account the change of the particle distribution showed that it is a very useful tool for estimating the actual bunch parameters evolution in the machine. The contribution of effects such as betatron coupling, noise and electron-cloud, to the emittance growth are planned to be included in the simulation code, in order to complement the existing semianalytical emittance evolution model.

\section{APPENDIX A: THE Q-GAUSSIAN DISTRIBUTION FUNCTION}

The q-Gaussian [17] which is used to describe more accurately bunch profiles with tails that differ from the ones of a normal distribution, has a probability density function given by:

$$
f(x)=\frac{\sqrt{\beta^{q G}}}{C_{q}} e_{q}\left(-\beta^{q G} x^{2}\right) .
$$

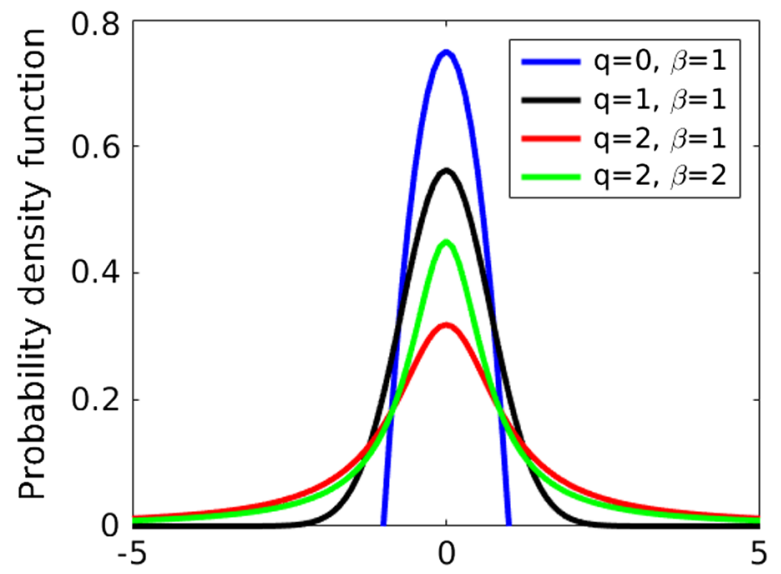

FIG. 21. The q-Gaussian distribution function for different $q$ and $\beta^{q G}$ values.
The q-exponential function is given by:

$e_{q}(x)= \begin{cases}\exp (x), & \text { if } q=1 \\ (1+(1-q) x)^{\frac{1}{1-q}}, & \text { if } q \neq 1 \text { and }(1+(1-q) x)>0 . \\ 0, & \text { if } q \neq 1 \text { and }(1+(1-q) x) \leq 0\end{cases}$

The parameter $q$ describes the weight of the tails, in the sense that the larger its value, the heavier the tails become, as presented in Fig. 21. In the limit of $q \rightarrow 1$, the normal distribution is obtained. The distribution is characterized as "light" tailed when $q<1$ and as "heavy" tailed when $q>1$. The normalization factor $C_{q}$ differs for specific ranges of the $q$ parameter and it is written as:

$$
C_{q}=\left\{\begin{array}{ll}
\frac{2 \sqrt{\pi}}{(3-q) \sqrt{1-q}} \frac{\Gamma\left(\frac{1}{1-q}\right)}{\Gamma\left(\frac{3-q}{2(1-q)}\right)}, & \text { for }-\infty<q<1 \\
\sqrt{\pi}, & \text { for } q=1 \\
\frac{\sqrt{\pi}}{\sqrt{q-1}} \frac{\Gamma\left(\frac{3-q}{2\left(\frac{1}{2}\right)}\right)}{\Gamma\left(\frac{1}{q-1}\right)}, & \text { for } 1<q<3
\end{array} .\right.
$$

The parameter $\beta^{q G}$ is a real positive number. As the normal distribution, the $q$-Gaussian is an even function taking its maximum at $x=0$, where

$$
f(0)=\frac{\sqrt{\beta^{q G}}}{C_{q}} .
$$

For a certain $q$ value, the higher is the value of $\beta^{q G}$, the larger is the maximum of the probability density function, as can be observed in Fig. 21. The standard deviation which also differs for specific ranges of the $q$ parameter, is given by:

$$
\sigma^{q G}= \begin{cases}\sqrt{\frac{1}{\beta^{q G}(5-3 q)}}, & \text { for } q<5 / 3 \\ \infty, & \text { for } 5 / 3 \leq q<2 \\ \text { undefined, }, & \text { for } 2 \leq q<3\end{cases}
$$

In the heavy tail regimes, the distribution is equivalent to the Student's t-distribution with a direct mapping between $q$ and the degrees of freedom $\nu$ [Eq. (A6)]. Statistically the q-Gaussian is a scaled reparametrization of the Student's $\mathrm{t}$-distribution [62] for which the parameter $\nu$ is constrained to be a positive integer related to the sample size. The advantage of the q-Gaussian function is that, by introducing the parameters $q$ and $\beta^{q G}$, a generalization of the Student's $\mathrm{t}$-distribution to negative and noninteger degrees of freedom is possible, where:

$$
q=\frac{\nu+3}{\nu+1} \quad \text { with } \quad \beta^{q G}=\frac{1}{3-q} .
$$




\section{APPENDIX B: LUMINOSITY CALCULATION FOR Q-GAUSSIAN DENSITY DISTRIBUTION FUNCTIONS}

Using Eq. (A1) as the probability density functions, the general luminosity formula in Eq. (1) is solved for q-Gaussian distributions in all planes. For the two beams being identical, integrating firstly over $s$ and $s_{0}$ :

$$
I_{s}^{q G}=\iint_{-\infty}^{\infty} \rho_{1 s}\left(s-s_{0}\right) \rho_{2 s}\left(s+s_{0}\right) d s d s_{0},
$$

and then, integrating over $\mathrm{x}$ and $\mathrm{y}$ :

$$
\begin{aligned}
I_{x y}^{q G} & =\iint_{-\infty}^{\infty} \rho_{1 x}(x) \rho_{1 y}(y) \rho_{2 x}(x) \rho_{2 y}(y) d x d y \\
& =\iint_{-\infty}^{\infty} \rho_{x}(x)^{2} \rho_{y}(y)^{2} d x d y,
\end{aligned}
$$

keeping in mind that for $w=x, y, s$ it is:

$$
\begin{aligned}
& w \in\left[ \pm \frac{1}{\sqrt{\beta^{q G}(1-q)}}\right], \quad \text { for }-\infty<q_{w}<1 \\
& w \in(-\infty, \infty), \quad \text { for } 1 \leq q_{w}<3,
\end{aligned}
$$

the solutions of these integrals are found to be:

$$
I_{s}^{q G}=1,
$$

and

$I_{x, y}^{q G}=\left\{\begin{array}{ll}\frac{\beta_{x, y}^{q G}}{C_{q_{x, y}}^{q G}} \frac{\sqrt{\pi} \Gamma\left(\frac{-3+q_{x, y}}{-1+q_{x, y}}\right)}{\sqrt{\beta_{x, y}^{q G}\left(1-q_{x, y}\right)} \Gamma\left(\frac{3 q_{x, y}-7}{2\left(-1+q_{x, y}\right)}\right)}, & \text { for }-\infty<q_{x, y}<1 \\ \frac{\beta_{x, y}^{q G}}{C_{q_{x, y}}^{q G}} \frac{\sqrt{\pi} \Gamma\left(\frac{-q_{x, y}+5}{2\left(-1+q_{x, y}\right)}\right)}{\left.\sqrt{\beta_{x, y}^{q G}\left(-1+q_{x, y}\right)}\right) \Gamma\left(\frac{2}{-1+q_{x, y}}\right)}, & \text { for } 1 \leq q_{x, y}<3\end{array}\right.$,

for $I_{x}^{q G} I_{y}^{q G}=I_{x y}^{q G}$ and, for $\beta_{x y}^{q G}$ and $C_{q_{x y}}$ being the beta parameters and the normalization factors in the transverse plane. After some simplifications, using also Eq. (A3) and Eq. (A5), it is found that the luminosity for q-Gaussian distribution functions depends on the $\mathcal{I}_{x, y}^{q G}$ [see Eq. (4)] which are defined as:

$\mathcal{I}_{x, y}^{q G}=\left\{\begin{array}{ll}\frac{(2+1 / k)^{2}}{2 \sqrt{3+2 k}} \frac{\Gamma(1+2 k) \Gamma(1 / 2+k)^{2}}{\Gamma(3 / 2+2 k) \Gamma(k)^{2}}, & \text { for }-\infty<q_{x, y}<1 \\ \frac{2}{\sqrt{-(3+2 k)}} \frac{\Gamma(-1 / 2-2 k) \Gamma(-k)^{2}}{\Gamma(-2 k) \Gamma(-1 / 2-k)^{2}}, & \text { for } 1 \leq q_{x, y}<\frac{5}{3}\end{array}\right.$,

for $k=\frac{1}{1-q_{x, y}}$. As for the Gaussian case [Eq. (2)], the luminosity for q-Gaussian beams colliding head-on does not depend on the longitudinal beam size.

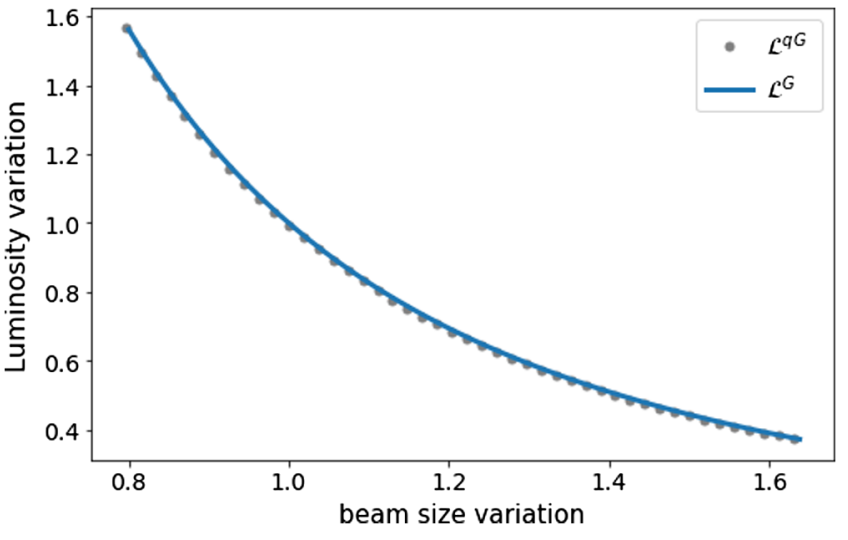

FIG. 22. Luminosity variation with respect to the transverse beam size variation, for the q-Gaussian $\left(\mathcal{L}^{q G}\right)$ with $q=1$ (i.e., normal distribution shape) and the Gaussian case $\left(\mathcal{L}^{G}\right)$, for the same beam parameters.

In Fig. 22, the variation of the luminosity is plotted with respect to the transverse beam size for the $q$-Gaussian case $\left(\mathcal{L}^{q G}\right)$ with $q=1$ (i.e., normal distribution shape) and the Gaussian case $\left(\mathcal{L}^{G}\right)$. Basically, the transverse beam sizes in Eq. (2) and Eq. (4) are being varied equivalently and the resulted luminosity changes are found using these two equations. The excellent agreement demonstrates that in the limit of $q \rightarrow 1$, the luminosity estimation for $q$-Gaussian distributions [given in Eq. (4)] allows to obtain the exact same result as for Gaussian distributions. By keeping the $q$ parameter constant (here $q=1$ ) and varying the beam size, the $\beta^{q G}$ parameter also varies.

[1] F. Antoniou, M. Hostettler, G. Iadarola, S. Papadopoulou, Y. Papaphilippou, D. Pellegrini, and G. Trad, Can we predict luminosity? in Proceedings, 7th Evian Workshop on LHC beam operation: Evian Les Bains, France, December 13-15, 2016, CERN (CERN, Geneva, 2017), pp. 125-132.

[2] S. Papadopoulou, F. Antoniou, I. Efthymiopoulos, M. Hostettler, G. Iadarola, N. Karastathis, S. Kostoglou, Y. Papaphilippou, and G. Trad, Monitoring and modelling of the LHC emittance and luminosity evolution in 2018, Proceedings, 10th International Particle Accelerator Conference (IPAC2019) (JACoW Publishing, Geneva, Switzerland, 2019), WEPTS046. p. 4, https://doi.org/ 10.18429/JACoW-IPAC2019-WEPTS046.

[3] S. Papadopoulou, Update on luminosity model including coupling, noise and burn-off for the emittance growth prediction, presented at the 158th HiLumi WP2 Meeting (CERN, Geneva, Switzerland, 2019).

[4] J. D. Bjorken and S. K. Mtingwa, Intrabeam scattering, Part. Accel. 13, 115 (1982), https://inspirehep.net/ literature/180068.

[5] A. Vivoli and M. Martini, Intra-beam scattering in the CLIC Damping Rings, Tech. Reps. No. CERN-ATS-2010094, CLIC-Note-834, 2010. 
[6] M. Martini, F. Antoniou, and Y. Papaphilippou, Intrabeam Scattering, ICFA Beam Dyn. Newsl. 69, 38 (2016), https://inspirehep.net/literature/1507570.

[7] A. Vivoli and M. Martini, Effects of Intrabeam Scattering and Synchrotron Radiation Damping when Reducing Transverse Emittances to Augment the LHC Luminosity, Tech. Reps. No. sLHC-PROJECT-Report0032, CERN-sLHC-PROJECT-Report-0032 (CERN, Geneva, 2010).

[8] R. Garoby, New RF exercises envisaged in the CERN-PS for the antiprotons production beam of the ACOL machine, Tech. Rep. No. CERN-PS-85-36-RF, 1985.

[9] I. B. Alonso, L. Rossi et al., HiLumi LHC Technical Design Report: Deliverable: D1.10, Tech. Report No. CERN-ACC-2015-0140, 2015.

[10] G. Apollinari, O. Brüning, T. Nakamoto, and L. Rossi, Chapter 1: High Luminosity Large Hadron Collider HLLHC. High Luminosity Large Hadron Collider HL-LHC, CERN Yellow Report No. CERN-2015-005, 1, 2017, chapter in High-Luminosity Large Hadron Collider (HL-LHC): Preliminary Design Report.

[11] W. Herr and B. Muratori, Concept of luminosity, in Intermediate accelerator physics, Proceedings, CERN Accelerator School, Zeuthen, Germany, September 15-26, 2003 (2003), pp. 361-377, https://doi.org/10.5170/CERN2006-002.361.

[12] M. Fitterer, G. Stancari, A. Valishev, R. Bruce, S. Papadopoulou, G. Papotti, D. Pellegrini, S. Redaelli, G. Trad, D. Valuch, G. Valentino, J. Wagner, and C. Xu, Effect of a resonant excitation on the evolution of the beam emittance and halo population, Tech. Report No. CERNACC-NOTE-2017-0037, 2017.

[13] S. Papadopoulou, F. Antoniou, T. Argyropoulos, M. Fitterer, M. Hostettler, and Y. Papaphilippou, Modeling and measurements of bunch profiles at the LHC, J. Phys. Conf. Ser. 874, 012008 (2017).

[14] H. Timko, P. Baudrenghien, J.E. Müller, and E. Shaposhnikova, Operational and beam dynamics aspects of the RF system in 2015, in Proceedings, 6th Evian Workshop on LHC beam operation: Evian Les Bains, France, December 15-17, 2015, CERN (CERN, Geneva, 2016), pp. 143-148.

[15] M. Kuhn, Emittance preservation at the LHC, Master's thesis, Hamburg U., 2013.

[16] P. Baudrenghien and T. Mastoridis, Longitudinal emittance blowup in the large hadron collider, Nucl. Instrum. Methods Phys. Res., Sect. A 726, 181 (2013).

[17] E. M.F. Curado and C. Tsallis, Generalized statistical mechanics: connection with thermodynamics, J. Phys. A 25, 1019 (1992).

[18] C. Tsallis, F. Baldovin, R. Cerbino, and P. Pierobon, Introduction to nonextensive statistical mechanics and thermodynamics, arXiv:cond-mat/0309093.

[19] I. Eftymiopoulos, LHC lumi days 2019, presented at the LHC Lumi Days 2019 Workshop, June 2019 (CERN, Geneva, Switzerland, 2019).

[20] H. G. Hereward, How good is the R.M.S. as a measure of beam size ?, Tech. Report No. CERN-MPS-DL-69-15 (CERN, Geneva, 1969).
[21] K. Potter, Luminosity Measurements and Calculations, in Proc. of the CERN Accelerator School, CAS 1992, CERN yellow report (1992), p. 117, https://doi.org/10.5170/ CERN-1994-001.117.

[22] G. Aad et al. (ATLAS Collaboration), Observation of a new particle in the search for the Standard Model Higgs boson with the ATLAS detector at the LHC, Phys. Lett. B 716, 1 (2012).

[23] S. Chatrchyan et al. (CMS Collaboration), Observation of a new boson at a mass of $125 \mathrm{GeV}$ with the CMS experiment at the LHC, Phys. Lett. B 716, 30 (2012).

[24] F. Antoniou and F. Zimmermann, Revision of intrabeam scattering with non-ultrarelativistic corrections and vertical dispersion for MAD-X, Tech. Report No. CERN-ATS2012-066 (CERN, Geneva, 2012).

[25] K. L.F. Bane, Intra-beam scattering, impedence, and instabilities in ultimate storage rings (2012), https://www.semanticscholar.org/paper/Intra-BeamScattering\%2C-Impedance\%2C-and-Instabilities-Bane/ 2445e07bf96b711768d9a3ab67fd7e7969423aa6.

[26] L. K. Spentzouris, C. Bhat, and P. L. Colestock, Measurements of Intrabeam Scattering Rates below Transition in the Fermilab Antiproton Accumulator, in Proceedings of the 18th Particle Accelerator Conference, New York, 1999 (IEEE, New York, 1999).

[27] W. Fischer, M. Bai, M. Blaskiewicz, J. M. Brennan, P. Cameron, R. Connolly, A. Lehrach, G. Parzen, S. Tepikian, K. Zeno, and J. van Zeijts, Measurements of intra-beam scattering growth times with gold beam below transition in rhic, in PACS2001. Proceedings of the 2001 Particle Accelerator Conference (Cat. No.01CH37268), Vol. 4 (IEEE, Piscataway, NJ, 2001), pp. 2857-2859.

[28] A. Piwinski, Intra-beam scattering, in Proceedings of the 9th International Conference on High Energy Accelerators: Stanford, USA (1974).

[29] M. Martini, Intrabeam scattering in the ACOL-AA machines, Tech. Report No. CERN-PS-84-9-AA (CERN, Geneva, 1984).

[30] K. L. F. Bane, H. Hayano, K. Kubo, T. Naito, T. Okugi, and J. Urakawa, Intrabeam scattering analysis of measurements at KEK's ATF damping ring, Phys. Rev. Accel. Beams 5, 084403 (2002).

[31] K. Kubo, S. K. Mtingwa, and A. Wolski, Intrabeam scattering formulas for high energy beams, Phys. Rev. Accel. Beams 8, 081001 (2005).

[32] V. Lebedev, Single and multiple intrabeam scattering in hadron colliders, AIP Conf. Proc. 773, 440 (2005).

[33] A. Piwinski, Intra-beam scattering, in Proceedings of the 9th International Conference on High Energy Accelerators: Stanford, USA (1974), https://doi.org/10.5170/ CERN-1992-001.226.

[34] J. M. Jowett, Introductory statistical mechanics for electron storage rings, AIP Conf. Proc. 153, 864 (1986), https:// inspirehep.net/literature/237003.

[35] J. Wei, A. Fedotov, W. Fischer, N. Malitsky, G. Parzen, and J. Qiang, Intrabeam scattering theory and rhic experiments, AIP Conf. Proc. 773, 389 (2005).

[36] G. Parzen, Intrabeam scattering growth rates for a bigaussian beam, arXiv:physics/0410028. 
[37] A. V. Fedotov, A. O. Sidorin, and A. V. Smirnov, IBS for non-gaussian distributions, in Proceedings of HB2010, Morschach, Switzerland (MOPD09) (2010), pp. 62-66, http://www.bnl.gov/isd/documents/74305.pdf.

[38] A. Xiao and M. Borland, Intrabeam scattering effect calculated for a non-gaussian-distributed linac beam, in Proceedings of PAC09, Vancouver, BC, Canada (IEEE, Piscataway, NJ, 2009).

[39] M. Venturini, Intrabeam scattering and wake field effects in low emittance electron rings, in Proceedings of the 19th Particle Accelerator Conference, Chicago, IL, 2001 (Cat. No. 01CH37268), Vol. 4 (IEEE, Piscataway, NJ, 2001), pp. 2958-2960.

[40] Accelerator Physics at the Tevatron Collider, edited by V. Lebedev and V. Shiltsev, Particle Acceleration and Detection (Springer, New York, 2014).

[41] M. Biagini, M. Boscolo, T. Demma, A. W. Chao, K. L. F. Bane et al., Multiparticle simulation of intrabeam scattering for SuperB, Conf. Proc. C110904, 2259 (2011), https:// inspirehep.net/literature/1079486.

[42] M. T. F. Pivi, CMAD: A self-consistent parallel code to simulate the electron cloud build-up and instabilities, Tech. Report No. SLAC-PUB-12970 (SLAC, Stanford, CA, 2007).

[43] K. G. Sonnad, F. Antoniou, Y. Papaphilippou, K. S. B. Li, M. Boscolo et al., Multi-particle simulation codes implementation to include models of a novel single-bunch feedback system and intra-beam scattering, Conf. Proc. C1205201, 3147 (2012), https://inspirehep.net/literature/ 1126820.

[44] P. Zenkevich, A. Bolshakov, and O. Boine-Frankenheim, Kinetic effects in multiple intra-beam scattering, AIP Conf. Proc. 773, 425 (2005).

[45] P. Zenkevich, O. Boine-Frankenheim, and A. Bolshakov, A new algorithm for the kinetic analysis of intra-beam scattering in storage rings, Nucl. Instrum. Methods Phys. Res., Sect. A 561, 284 (2006).

[46] T. O. Raubenheimer, The Generation and acceleration of low emittance flat beams for future linear colliders, Reports No. SLAC-0387, No. SLAC-387, No. UMI-92-17870, No. SLAC-R-0387, No. SLAC-R-387, 1991.

[47] K. L. F. Bane, A Simplified model of intrabeam scattering, in Particle accelerator. Proceedings, 8th European Conference, EPAC 2002, Paris, France, June 3-7, 2002 (EPS-IGA and CERN, Geneva, 2002), pp. 1443-1445, arXiv:physics/0206002.

[48] F. Antoniou, Optics design of intrabeam scattering dominated damping rings, Ph.D. thesis, National (Metsovian) Technical University of Athens, Athens, 2012.
[49] Y. Papaphilippou et al., Conceptual design of the CLIC damping rings, Tech. Report No. CERN-ATS-2012-176 (CERN, Geneva, 2012).

[50] M. Lamont and O. Johnson, LHC beam and luminosity lifetimes revisited, Tech. Report No. CERN-ACC-20140255 (CERN, Geneva, 2014).

[51] E. Métral et al., Update of the HL-LHC operational scenarios for proton operation, Tech. Report No. CERNACC-NOTE-2018-0002 (CERN, Geneva, 2018).

[52] MAD-X homepage, http://mad.web.cern.ch/mad/.

[53] S. Burger, A. Boccardi, E. Bravin, A. Goldblatt, A. Ravni, F. Roncarolo, and R. Sautier, Turn by Turn Profile Monitors for the CERN SPS and LHC, Tech. Report No. CERN-ACC-2013-0299, 2013.

[54] M. Sapinski, W. Andreazza, B. Dehning, A. Guerrero, M. Patecki, and R. Versteegen, The first experience with LHC beam gas ionization monitor, Tech. Report No. CERNATS-2012-286 (CERN, Geneva, 2012).

[55] J. Bosser, J. Camas, L. Evans, G. Ferioli, R. Hopkins, J. Mann, and O. Olsen, Transverse emittance measurement with a rapid wire scanner at the CERN SPS, Nucl. Instrum. Methods Phys. Res., Sect. A 235, 475 (1985).

[56] F. Méot, L. Ponce, J. Bosser, and R. Jung, Diagnostic with synchrotron radiation of the LHC proton beams, in Particle accelerator. Proceedings, 8th European Conference, EPAC 2002, Paris, France, June 3-7, 2002 (EPS-IGA and CERN, Geneva, 2002), pp. 1945-1947.

[57] G. Papotti, T. Bohl, F. Follin, and U. Wehrle, Longitudinal beam measurements at the LHC: The LHC beam quality monitor, Tech. Report No. CERN-ATS-2011-220, 2011.

[58] T. Bohl and J. F. Malo, The APWL wideband wall current monitor, Tech. Report No. CERN-BE-2009-006 (CERN, Geneva, 2009).

[59] A. Jeff, M. Andersen, A. Boccardi, S. Bozyigit, E. Bravin, T. Lefevre, A. Rabiller, F. Roncarolo, C. P. Welsch, and A. S. Fisher, Longitudinal density monitor for the LHC, Phys. Rev. Accel. Beams 15, 032803 (2012).

[60] S. Papadopoulou, Bunch characteristics evolution for lepton and hadron rings under the influence of the Intrabeam scattering effect, Ph.D. thesis, University of Crete, Greece, 2019.

[61] J. E. Müller, Longitudinal intensity effects in the CERN Large Hadron Collider, Ph.D. thesis, Ecole Polytechnique, Lausanne, 2016, https://inspirehep.net/literature/1503638.

[62] Student, The probable error of a mean, Biometrika 6, 1 (1908). 